\title{
Covariant quiver gauge theories
}

\author{
Richard J. Szabo ${ }^{a, b, c}$ and Omar Valdivia ${ }^{a, b, c, d}$ \\ ${ }^{a}$ Department of Mathematics, Heriot-Watt University, \\ Colin Maclaurin Building, Riccarton, Edinburgh EH14 4AS, U.K. \\ ${ }^{b}$ Maxwell Institute for Mathematical Sciences, \\ Edinburgh, U.K. \\ ${ }^{c}$ The Tait Institute, \\ Edinburgh, U.K. \\ ${ }^{d}$ Departamento de Física, Universidad de Concepción, \\ Casilla 160-C, Concepción, Chile \\ E-mail: R.J.Szabo@hw.ac.uk, ov32@hw.ac.uk
}

ABSTRACT: We consider dimensional reduction of gauge theories with arbitrary gauge group in a formalism based on equivariant principal bundles. For the classical gauge groups we clarify the relations between equivariant principal bundles and quiver bundles, and show that the reduced quiver gauge theories are all generically built on the same universal symmetry breaking pattern. The formalism enables the dimensional reduction of Chern-Simons gauge theories in arbitrary odd dimensionalities. The reduced model is a novel Chern-Simons-Higgs theory consisting of a Chern-Simons term valued in the residual gauge group plus a higher order gauge and diffeomorphism invariant coupling of Higgs fields with the gauge fields. We study the moduli spaces of solutions, which in some instances provide geometric representations of certain quiver varieties as moduli spaces of flat invariant connections. As physical applications, we consider dimensional reductions involving non-compact gauge supergroups as a means for systematically inducing novel couplings between gravity and matter. In particular, we reduce Chern-Simons supergravity to a quiver gauge theory of AdS gravity involving a non-minimal coupling to scalar Higgs fermion fields.

Keywords: Chern-Simons Theories, Gauge Symmetry, Classical Theories of Gravity, Differential and Algebraic Geometry

ARXIV EPRINT: 1404.4319 


\section{Contents}

1 Introduction and summary 1

2 Equivariant dimensional reduction $\quad 3$

2.1 Equivariant principal bundles 3

2.2 Invariant connections 5

$\begin{array}{ll}2.3 & \text { Dimensional reduction of Yang-Mills theory }\end{array}$

$\begin{array}{lll}3 & \text { Principal quiver bundles } & 7\end{array}$

4 Covariant quiver gauge theories $\quad 10$

$\begin{array}{lll}\text { 4.1 Chern-Simons theory and transgression forms } & 10\end{array}$

$\begin{array}{lll}4.2 & \text { Topological Chern-Simons-Higgs models } & 12\end{array}$

$\begin{array}{lll}4.3 \text { Moduli spaces of solutions } & 14\end{array}$

5 Quiver gauge theory of AdS gravity $\quad \mathbf{1 8}$

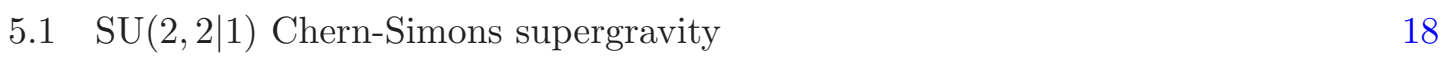

$\begin{array}{ll}5.2 \text { Dimensional reduction } & 19\end{array}$

5.3 Applications 23

$\begin{array}{ll}\text { A Classical gauge groups } & 24\end{array}$

B Extended Cartan homotopy formula $\quad \mathbf{2 5}$

B.1 Restricted homotopy formula 25

B.2 Chern-Weil theorem 26

$\begin{array}{ll}\text { B.3 Triangle equation } & 26\end{array}$

$\begin{array}{ll}\mathrm{C} \operatorname{SU}(2,2 \mid N) \text { Chern-Simons supergravity } & 27\end{array}$

$\begin{array}{ll}\text { C.1 Supergravity Lagrangian } & 27\end{array}$

$\begin{array}{lll}\text { C.2 Representation of } \mathfrak{s u}(2,2 \mid 1) & 28\end{array}$

\section{Introduction and summary}

Dimensional reduction provides a means of unifying gauge and Higgs sectors into a pure Yang-Mills theory in higher dimensions. The reductions are particularly rich if the extra spacetime dimensions admit isometries, which can then be implemented on gauge orbits of fields [1]. The natural setting for spacetime isometries are coset spaces $G / H$ of compact Lie groups in which Yang-Mills theory on the product space $M \times G / H$ is reduced to a Yang-Mills-Higgs theory on the manifold $M$; the construction can be extended supersymmetrically and also embedded in string theory [2]. Equivariant dimensional reduction is 
an alternative approach which naturally incorporates background fluxes coming from the topology of the canonical connections on the principal $H$-bundle $G \rightarrow G / H[3-5]$; the reduced Yang-Mills-Higgs model is then succinctly described by a quiver gauge theory on $M$ whose underlying quiver is canonically associated to the representation theory of the Lie groups $H \subset G$. Such reductions have been used to describe vortices as generalized instantons in higher-dimensional Yang-Mills theory [6-10], as well as to construct explicit $\mathrm{SU}(2)$-equivariant monopole and dyon solutions of pure Yang-Mills theory in four dimensions [11].

A related approach is described in [12] which systematically translates the inverse relations of restriction and induction of vector bundles [3] into the framework of principal bundles. In this formulation there is no restriction on the structure group and it permits, for instance, the application of equivariant dimensional reduction techniques to gauge theories involving arbitrary gauge groups $\mathcal{G}$. In the following we adapt such an approach to the simplest case where $G=\mathrm{SU}(2)$ and $H=\mathrm{U}(1)$, so that the internal coset space $G / H$ is the two-sphere $S^{2}$ or the complex projective line $\mathbb{C} P^{1}$. This example turns out to be rich enough to capture many of the general features that one would encounter on generic cosets $G / H$.

The geometric structures arising from reductions of SU(2)-invariant Yang-Mills theory have been thoroughly studied in a multitude of different contexts [13-15], while coset space dimensional reduction of five-dimensional Chern-Simons theory with gauge group $\mathcal{G}=\mathrm{SU}(2)$ is considered in [16] and some physical characteristics of its moduli space of solutions are pointed out. In this paper we pursue the equivariant dimensional reduction of topological gauge theories. We study the related problems of generalizing equivariant dimensional reduction to arbitrary gauge groups $\mathcal{G}$ and extending these techniques to Chern-Simons gauge theories. We classify the symmetry breaking patterns induced by $G$-invariant connections whose gauge group $\mathcal{G}$ lies in one of the four infinite families of classical Lie groups. We show that the unbroken gauge group of the reduced theory is generically the same (without any conditions on the background Dirac monopole charges) in all cases. As a consequence, the induced quiver gauge theories are the same for any classical gauge group (up to redefinitions of the coupling constants); in our approach many of the geometric ingredients used in [3-5] to derive these quiver gauge theories are translated into an algebraic framework. We will then explore $G$-equivariant dimensional reduction of pure topological gauge theories. We calculate the reduction of an arbitrary odd-dimensional Chern-Simons form over $\mathbb{C} P^{1}$; although Chern-Simons Lagrangians are not gauge-invariant, we circumvent this problem by regarding them in the framework of transgression forms. The reduced theory is a novel diffeomorphism-invariant Chern-Simons-Higgs model, which can have local degrees of freedom whose dynamics and canonical structure are rather delicate to disentangle; our generally covariant models are therefore generically not topological field theories.

As mathematical applications, we study the moduli spaces of classical solutions of these field theories and obtain some geometric interpretations of representation theoretic results. For example, we describe the quiver varieties parameterizing semisimple representations of certain deformed preprojective algebras as moduli spaces of $\mathrm{SU}(2)$-invariant flat 
$\mathcal{G}$-connections on the three-manifold $\mathcal{M}=\mathbb{R} \times S^{2}$. As physical applications, we consider the case of non-compact gauge supergroups. In particular, we perform the dimensional reduction of five-dimensional Chern-Simons supergravity over $\mathbb{C} P^{1}$. We show that if the Higgs fields are bifundamental fields in the fermionic sector of the gauge algebra, then the reduced action contains the standard Einstein-Hilbert term plus a non-minimal coupling of the Higgs fermions to the curvature. This reduction scheme thus constitutes a novel systematic way to couple scalar fermionic fields to gravitational Lagrangians, in a manner whereby non-vacuum solutions of three-dimensional AdS gravity can be lifted to give new solutions of five-dimensional supergravity on product spacetimes $M \times S^{2}$.

This paper is organized as follows. In section 2 we discuss general aspects of SU(2)equivariant dimensional reduction and revisit the example of pure Yang-Mills theory as illustration. In section 3 the symmetry breaking patterns are analysed for the classical gauge groups and the geometric structure of general principal quiver bundles is described. In section 4 we derive the SU(2)-equivariant dimensional reduction of Chern-Simons gauge theories in arbitrary odd dimensionality and discuss some explicit examples. In section 5 we carry out the dimensional reduction of five-dimensional Chern-Simons supergravity and point out some possible implications. Three appendices at the end of the paper contain some technical details which are used in the calculations of the main text: in appendix A we summarise the pertinent group theory data for the classical gauge groups, in appendix B we explain the extended Cartan homotopy formula and some of its corollaries, and in appendix $\mathrm{C}$ we describe the structure of Chern-Simons supergravity based on the supergroup $\mathrm{SU}(2,2 \mid N)$.

\section{Equivariant dimensional reduction}

\subsection{Equivariant principal bundles}

In this paper we study gauge theories on the product space $\mathcal{M}=M \times S^{2}$. Here $M$ is a closed $d$-dimensional manifold with local coordinates $\left(x^{\mu}\right)_{\mu=1}^{d}$. On the sphere $S^{2} \simeq \mathbb{C} P^{1}$ we use complex coordinates $(y, \bar{y})$ defined by stereographic parameterization. We identify $S^{2}$ with the coset space $\mathrm{SU}(2) / \mathrm{U}(1)$. This induces a transitive action of $\mathrm{SU}(2)$ on $S^{2}$ which we extend to the trivial action on $M$. In order to obtain dimensionally reduced gauge invariant field theories starting from arbitrary gauge groups $\mathcal{G}$, in this section we study $\mathrm{SU}(2)$-equivariant principal bundles on $\mathcal{M}$ and their corresponding $\mathrm{SU}(2)$-invariant connections. We follow for a large part the treatment of [12].

Every $\mathrm{SU}(2)$-equivariant principal bundle over $S^{2}$ with structure group $\mathcal{G}$ is isomorphic to a quotient space [17]

$$
\mathcal{P}_{\rho}=\mathrm{SU}(2) \times{ }_{\rho} \mathcal{G}
$$

where $\rho: \mathrm{U}(1) \rightarrow \mathcal{G}$ is a homomorphism and the elements of $\mathrm{SU}(2) \times_{\rho} \mathcal{G}$ are equivalence classes $[s, g]$ on $\mathrm{SU}(2) \times \mathcal{G}$ with respect to the equivalence relation

$$
(s, g) \equiv\left(s s_{0}, \rho\left(s_{0}\right)^{-1} g\right)
$$


for all elements $s_{0} \in \mathrm{U}(1) \subset \mathrm{SU}(2)$. The bundle projection $\pi: \mathcal{P}_{\rho} \rightarrow S^{2}$ is given by

$$
\pi([s, g])=[s]
$$

where $[s]$ denotes the left coset $s \cdot \mathrm{U}(1)$ in $\mathrm{SU}(2)$. Bundles $\mathcal{P}_{\rho}, \mathcal{P}_{\rho^{\prime}}$ are isomorphic if and only if the homomorphisms $\rho, \rho^{\prime}: \mathrm{U}(1) \rightarrow \mathcal{G}$ take values in the same conjugacy class of $\mathcal{G}$.

Let $P$ be an SU(2)-equivariant principal $\mathcal{G}$-bundle over $\mathcal{M}=M \times S^{2}$ and select a good open covering $\left\{U_{i}\right\}_{i \in I}$ of $M$, i.e. all $U_{i}$ are contractible. Then the restrictions $\left.P\right|_{U_{i} \times S^{2}}$ are $\mathrm{SU}(2)$-equivariant bundles which are trivial on each $U_{i}$, so that

$$
\left.P\right|_{U_{i} \times S^{2}} \simeq U_{i} \times \mathcal{P}_{\rho_{i}}
$$

where the homomorphisms $\rho_{i}: \mathrm{U}(1) \rightarrow \mathcal{G}$ may be different for every open set $U_{i} \subset M$. However, on the non-empty intersections $U_{i j}=U_{i} \cap U_{j}$ in $M$, the restrictions $\left.P\right|_{U_{i j} \times S^{2}}$ are isomorphic to

$$
U_{i j} \times\left.\mathcal{P}_{\rho_{j}} \simeq P\right|_{U_{i j} \times S^{2}} \simeq U_{i j} \times \mathcal{P}_{\rho_{i}} .
$$

This means that $\mathcal{P}_{\rho_{j}} \simeq \mathcal{P}_{\rho_{i}}$ and hence $\rho_{i}, \rho_{j}$ take values in the same conjugacy class of $\mathcal{G}$. If $M$ is connected, a representative homomorphism $\rho$ can be chosen such that

$$
\left.P\right|_{U_{i} \times S^{2}} \simeq U_{i} \times \mathcal{P}_{\rho}
$$

for all $i \in I$, and which satisfies

$$
\rho=h_{i j}^{-1} \rho h_{i j}
$$

for all transition functions $h_{i j}: U_{i j} \rightarrow \mathcal{G}$. This implies that $h_{i j}$ take values in the centralizer of the image $\rho(\mathrm{U}(1))$ in $\mathcal{G}$, which we denote by

$$
\mathcal{H}=Z_{\mathcal{G}}(\rho(\mathrm{U}(1))) .
$$

Thus the collection of transition functions $\left\{h_{i j}\right\}$ for $i, j \in I$ defines a principal bundle $P_{M}$ over $M$ with structure group $\mathcal{H}$ which is the residual gauge group after dimensional reduction.

The homomorphism $\rho$ is determined by specifying a unique element $\Lambda \in \mathfrak{g}$, where $\mathfrak{g}$ is the Lie algebra of $\mathcal{G}$. For this, introduce the Pauli spin matrices

$$
\sigma_{1}=\left(\begin{array}{ll}
0 & 1 \\
1 & 0
\end{array}\right), \quad \sigma_{2}=\left(\begin{array}{cc}
0 & -\mathrm{i} \\
\mathrm{i} & 0
\end{array}\right), \quad \sigma_{3}=\left(\begin{array}{cc}
1 & 0 \\
0 & -1
\end{array}\right)
$$

so that $T_{a}=-\frac{\mathrm{i}}{2} \sigma_{a}$ for $a=1,2,3$ generate the defining representation of the Lie algebra $\mathfrak{s u}(2)$, where the $\mathrm{U}(1)$ subgroup of $\mathrm{SU}(2)$ is generated by $T_{3}$. Any element of $\mathrm{U}(1)$ can be written as $\exp \left(t T_{3}\right)$, where $t \in \mathbb{R}$, and the image of this element under the homomorphism $\rho$ is

$$
\rho\left(\exp \left(t T_{3}\right)\right)=\exp (t \Lambda)
$$

where $\exp (t \Lambda) \in \mathcal{G}$. Note that the identity element of $\mathrm{U}(1) \subset \mathrm{SU}(2)$ corresponds to $t=4 \pi$, so that

$$
\exp \left(4 \pi T_{3}\right)=\mathbb{1}_{\mathrm{SU}(2)}
$$


and since $\rho$ is a homomorphism it follows that $\Lambda$ must satisfy

$$
\exp (4 \pi \Lambda)=\mathbb{1}_{\mathcal{G}} .
$$

This leads generally to an algebraic quantization condition on $\rho: \mathrm{U}(1) \rightarrow \mathcal{G}$ which we describe explicitly in what follows.

The operations of restriction and induction [3] work for principal bundles in the same way as for vector bundles. Given an SU(2)-equivariant principal bundle $P \rightarrow M \times S^{2}$, its restriction $\left.P\right|_{M \times\left[\mathbb{1}_{\mathrm{SU}(2)}\right]}$ defines a $\mathrm{U}(1)$-equivariant principal bundle on $M$ which is isomorphic to $P_{M}$. The U(1)-action on the fibre is defined by the homomorphism $\rho$ : $\mathrm{U}(1) \rightarrow \mathcal{G}$ and it extends trivially on the base space $M$. The inverse operation gives $P=\mathrm{SU}(2) \times\left.{ }_{\rho} P\right|_{M \times\left[\mathbb{1}_{\mathrm{SU}(2)}\right]}$.

\subsection{Invariant connections}

For a principal bundle $P$ over $\mathcal{M}$ with connection one-form $\omega \in \Omega^{1}(P, \mathfrak{g})$, a local gauge potential $\mathcal{A} \in \Omega^{1}(\mathcal{U}, \mathfrak{g})$ on a contractible open subset $\mathcal{U} \subset \mathcal{M}$ is obtained via a local section $\sigma: \mathcal{U} \rightarrow P$ as the pull-back

$$
\mathcal{A}=\sigma^{*} \omega
$$

Let $\omega$ be an $\mathrm{SU}(2)$-invariant connection on $P \rightarrow \mathcal{M}$. In each open subset $U_{i} \subset M$, we can pull-back $\omega$ to an $\mathrm{SU}(2)$-invariant connection on $U_{i} \times \mathcal{P}_{\rho}$ which corresponds to a gauge potential $\mathcal{A}_{i}$ on $U_{i} \times S^{2}$ whose components are given by [12]

$$
\begin{aligned}
& \mathcal{A}_{i, \mu}=A_{i, \mu}, \\
& \mathcal{A}_{i, y}=\frac{-1}{1+y \bar{y}}\left(\mathrm{i} \bar{y} \Lambda+\Phi_{i}\right), \\
& \mathcal{A}_{i, \bar{y}}=\frac{1}{1+y \bar{y}}\left(\mathrm{i} y \Lambda+\Phi_{i}^{\dagger}\right),
\end{aligned}
$$

and are subjected to the invariance constraints

$$
\begin{aligned}
{\left[\Lambda, A_{i, \mu}\right] } & =0, \\
{\left[\Lambda, \Phi_{i}\right] } & =-\mathrm{i} \Phi_{i}, \\
{\left[\Lambda, \Phi_{i}^{\dagger}\right] } & =\mathrm{i} \Phi_{i}^{\dagger} .
\end{aligned}
$$

On non-empty overlaps $U_{i j} \subset M$ these fields obey the relations

$$
\begin{aligned}
& A_{j}=h_{i j}^{-1} A_{i} h_{i j}+h_{i j}^{-1} \mathrm{~d} h_{i j}, \\
& \Phi_{j}=h_{i j}^{-1} \Phi_{i} h_{i j},
\end{aligned}
$$

where $h_{i j}: U_{i j} \rightarrow \mathcal{H}$ are the transition functions of $P_{M}$, and $A_{i}=A_{i, \mu} \mathrm{d} x^{\mu}$. The collection of local gauge potentials $A_{i}$ defines a connection on $P_{M}$, and the constraints (2.17) imply that $A_{i}$ take values in the Lie algebra $\mathfrak{h}$ of $\mathcal{H}$ which is consistent with $P_{M}$ having $\mathcal{H}$ as structure group. The collection of local adjoint scalar fields $\Phi_{i}$ defines a section of the vector bundle $\operatorname{ad}\left(P_{M}\right):=P_{M} \times_{\text {ad }} \mathfrak{g}$ associated to $P_{M}$ by the adjoint representation of $\mathcal{H}$ on $\mathfrak{g}$. In the following we write $A, \Phi$ with $\left.A\right|_{U_{i}}=A_{i}$ and $\left.\Phi\right|_{U_{i}}=\Phi_{i}$. 


\subsection{Dimensional reduction of Yang-Mills theory}

On $\mathcal{M}=M \times S^{2}$ the metric is taken to be the direct product of a chosen metric $g_{\mu \nu}$ on $M$ and the round metric of the two-sphere, so that

$$
\mathrm{d} s^{2}=G_{\mu^{\prime} \nu^{\prime}} \mathrm{d} x^{\mu^{\prime}} \otimes \mathrm{d} x^{\nu^{\prime}}=g_{\mu \nu} \mathrm{d} x^{\mu} \otimes \mathrm{d} x^{\nu}+\frac{4 R^{2}}{(1+y \bar{y})^{2}} \mathrm{~d} y \otimes \mathrm{d} \bar{y}
$$

where the indices $\mu^{\prime}, \nu^{\prime}$ run over $1, \ldots, d+2$ and $R$ is the radius of $S^{2}$. For a principal $\mathcal{G}$-bundle $P \rightarrow \mathcal{M}$ with gauge potential $\mathcal{A}$, the Yang-Mills Lagrangian is given by

$$
L_{\mathrm{YM}}=-\frac{1}{4 g_{\mathrm{YM}}^{2}} \sqrt{G} \operatorname{Tr}\left(\mathcal{F}_{\mu^{\prime} \nu^{\prime}} \mathcal{F}^{\mu^{\prime} \nu^{\prime}}\right)
$$

where $\mathcal{F}$ is the curvature two-form

$$
\mathcal{F}=\mathrm{d} \mathcal{A}+\mathcal{A} \wedge \mathcal{A}=\frac{1}{2} \mathcal{F}_{\mu^{\prime} \nu^{\prime}} \mathrm{d} x^{\mu^{\prime}} \wedge \mathrm{d} x^{\nu^{\prime}}
$$

and $G=\operatorname{det}\left(G_{\mu^{\prime} \nu^{\prime}}\right)$. Here $g_{\mathrm{YM}}$ is the Yang-Mills coupling constant and $\operatorname{Tr}$ denotes a nondegenerate invariant quadratic form on the Lie algebra $\mathfrak{g}$ of the gauge group $\mathcal{G}$, which for $\mathcal{G}$ semisimple is proportional to the Killing-Cartan form.

Expanding (2.23) into components along $M$ and $\mathbb{C} P^{1}$ we get

$$
L_{\mathrm{YM}}=-\frac{1}{4 g_{\mathrm{YM}}^{2}} \sqrt{G} \operatorname{Tr}\left(\mathcal{F}_{\mu \nu} \mathcal{F}^{\mu \nu}+\frac{(1+y \bar{y})^{2}}{2 R^{2}} g^{\mu \nu}\left(\mathcal{F}_{\mu y} \mathcal{F}_{\nu \bar{y}}+\mathcal{F}_{\mu \bar{y}} \mathcal{F}_{\nu y}\right)+\frac{(1+y \bar{y})^{4}}{8 R^{4}} \mathcal{F}_{y \bar{y}} \mathcal{F}_{\bar{y} y}\right)
$$

where from (2.14)-(2.16) we have

$$
\begin{aligned}
& \mathcal{F}_{\mu \nu}=F_{\mu \nu}, \\
& \mathcal{F}_{\mu y}=-\frac{1}{1+y \bar{y}} \nabla_{\mu} \Phi, \\
& \mathcal{F}_{\mu \bar{y}}=\frac{1}{1+y \bar{y}} \nabla_{\mu} \Phi^{\dagger}, \\
& \mathcal{F}_{y \bar{y}}=\frac{1}{(1+y \bar{y})^{2}}\left(2 \mathrm{i} \Lambda-\left[\Phi, \Phi^{\dagger}\right]\right),
\end{aligned}
$$

with

$$
\begin{aligned}
F & =\mathrm{d} A+A \wedge A=\frac{1}{2} F_{\mu \nu} \mathrm{d} x^{\mu} \wedge \mathrm{d} x^{\nu}, \\
\nabla \Phi & =\mathrm{d} \Phi+[A, \Phi]=\nabla_{\mu} \Phi \mathrm{d} x^{\mu} .
\end{aligned}
$$

Integrating the corresponding Yang-Mills action

$$
S_{\mathrm{YM}}=\int_{\mathcal{M}} \mathrm{d}^{d+2} x \sqrt{G} L_{\mathrm{YM}}
$$

over $S^{2} \simeq \mathbb{C} P^{1}$ using

$$
\int_{\mathbb{C} P^{1}} \frac{R^{2}}{(1+y \bar{y})^{2}} \mathrm{~d} y \wedge \mathrm{d} \bar{y}=4 \pi R^{2}
$$


we get the action

$$
\begin{aligned}
S_{\mathrm{YMH}}=\frac{\pi R^{2}}{g_{\mathrm{YM}}^{2}} \int_{M} \mathrm{~d}^{d} x \sqrt{g} \operatorname{Tr}\left(F_{\mu \nu}\left(F^{\mu \nu}\right)^{\dagger}\right. & +\frac{1}{2 R^{2}}\left(\nabla_{\mu} \Phi \nabla^{\mu} \Phi^{\dagger}+\nabla_{\mu} \Phi^{\dagger} \nabla^{\mu} \Phi\right) \\
& \left.+\frac{1}{8 R^{4}}\left(2 \mathrm{i} \Lambda-\left[\Phi, \Phi^{\dagger}\right]\right)^{2}\right)
\end{aligned}
$$

which describes a Yang-Mills-Higgs theory on $M$ with gauge group $\mathcal{H}[1,12,15]$.

\section{Principal quiver bundles}

In order to solve the constraint equations (2.17)-(2.19) explicitly, it is necessary to fix the element $\Lambda \in \mathfrak{g}$ and therefore the gauge group $\mathcal{G}$. In this section we consider the case where $\mathcal{G}$ is one of the classical Lie groups $\mathrm{U}(n), \mathrm{SO}(2 n), \mathrm{SO}(2 n+1)$, or $\mathrm{Sp}(2 n)$. In this case equivariant dimensional reduction gives principal $\mathcal{H}$-bundles $P_{M} \rightarrow M$ which can be characterized in terms of quivers, and (2.34) becomes an action for a quiver gauge theory on $M$.

In the Cartan-Weyl basis, the generators of the gauge group $\mathcal{G}$ satisfy the commutation relations

$$
\begin{aligned}
{\left[H_{i}, H_{j}\right] } & =0 \\
{\left[H_{i}, X_{\alpha}\right] } & =\alpha_{i} X_{\alpha}, \\
{\left[X_{\alpha}, X_{\beta}\right] } & = \begin{cases}N_{\alpha, \beta} X_{\alpha+\beta} & \text { if } \alpha+\beta \text { is a root }, \\
0 & \text { otherwise, }\end{cases} \\
{\left[X_{\alpha}, X_{-\alpha}\right] } & =\frac{2}{|\alpha|^{2}} \sum_{i=1}^{n} \alpha_{i} H_{i},
\end{aligned}
$$

where $n$ is the rank of $\mathcal{G}$, the subset $\left\{H_{i}\right\}_{i=1}^{n}$ generates the Cartan subalgebra $\mathfrak{t} \subset \mathfrak{g}$, the vectors $\alpha$ are the roots of the Lie algebra $\mathfrak{g}$ of $\mathcal{G}$, and $\left\{X_{\alpha}\right\}$ are the root vectors with normalization constants $N_{\alpha, \beta}$. By gauge invariance, the element $\Lambda \in \mathfrak{g}$ can be conjugated into the Cartan subalgebra generated by $\left\{H_{i}\right\}$. Then there is still a residual gauge symmetry under the discrete Weyl subgroup $\mathcal{W} \subset \mathcal{G}$ which acts by permuting the eigenvalues $\lambda_{i}$, $i=1, \ldots, n$ of $\Lambda$. We can use this symmetry to group $\lambda_{i}$ into $m+1$ degenerate blocks, $0 \leq m \leq n-1$, of dimensions $k_{\ell}$ such that $\lambda_{k_{0}+k_{1}+\cdots+k_{\ell-1}+1}=\cdots=\lambda_{k_{0}+k_{1}+\cdots+k_{\ell-1}+k_{\ell}}=: \alpha_{\ell}$ for $\ell=0,1, \ldots, m$, where $k_{-1}:=0$ and

$$
\sum_{\ell=0}^{m} k_{\ell}=n .
$$

Then the element $\Lambda$ can be expanded as

$$
\Lambda=\mathrm{i} \sum_{\ell=0}^{m} \alpha_{\ell} \sum_{i=1}^{k_{\ell}} H_{k_{1}+\cdots+k_{\ell-1}+i} .
$$


Similarly, the Higgs fields $\Phi$ and the gauge field $A$ can both be expanded in the Cartan-Weyl basis as

$$
\begin{aligned}
& \Phi=\sum_{i=1}^{n} \phi_{i} H_{i}+\sum_{\alpha>0}\left(\phi_{\alpha} X_{\alpha}+\phi_{-\alpha} X_{-\alpha}\right), \\
& A=\sum_{i=1}^{n} A_{i} H_{i}+\sum_{\alpha>0}\left(A_{\alpha} X_{\alpha}+A_{-\alpha} X_{-\alpha}\right) .
\end{aligned}
$$

Let us first consider the unitary gauge group $\mathcal{G}=\mathrm{U}(n)$. Since $\Lambda \in \mathfrak{u}(n)$, it may be represented by a Hermitian $n \times n$ matrix which can always be taken to be diagonal by conjugation with a suitable element $g \in \mathrm{U}(n)$. The roots and the forms of the generators in the Cartan-Weyl basis are summarized in appendix A.

Using

$$
\left[H_{i}, X_{e_{j}-e_{k}}\right]=\left(\delta_{j i}-\delta_{k i}\right) X_{e_{j}-e_{k}}
$$

the invariance constraints (2.18) and (2.19) yield

$$
\phi_{i}=0, \quad \phi_{j k}\left(\lambda_{j}-\lambda_{k}+1\right)=0=\phi_{k j}\left(\lambda_{k}-\lambda_{j}+1\right) .
$$

To allow for non-trivial solutions, it is necessary to require $\lambda_{k}-\lambda_{j}= \pm 1$. Using Weyl symmetry to restrict attention to $\lambda_{j}-\lambda_{k}=-1$ with $\lambda_{j} \neq \lambda_{k} \neq 0$, we find $\phi_{k j}=0$ while $\phi_{j k}$ can be non-vanishing. However, not all of the fields $\phi_{j k}$ are non-zero. The only nonvanishing components arise when $j$ and $k$ belong to neighbouring blocks of indices. If $j, k$ belong to the same block $K_{(\ell)}:=\left\{k_{0}+k_{1}+\cdots+k_{\ell-1}+i\right\}_{i=1}^{k_{\ell}}$, then $\lambda_{j}=\lambda_{k}=\alpha_{\ell}$ and so $\phi_{j k}=0$ by (3.10). On the other hand, if $j \in K_{(\ell)}$ and $k \in K_{(\ell+1)}$, then $\lambda_{j}=\alpha_{\ell}$ and $\lambda_{k}=\alpha_{\ell+1}$, and by (3.10) if $\phi_{j k} \neq 0$ then $\alpha_{\ell}-\alpha_{\ell+1}=-1$, so we have $\alpha_{\ell}=\alpha+\ell$ for $\ell=0,1, \ldots, m$ and $\alpha:=\alpha_{0}$. Therefore the Higgs field (3.7) has the form

$$
\Phi=\sum_{\ell=0}^{m} \phi_{(\ell+1)}
$$

where

$$
\phi_{(\ell+1)}=\sum_{\substack{j \in K_{(\ell)}, k \in K_{(\ell+1)} \\ j<k}} \phi_{j k} X_{e_{j}-e_{k}}
$$

with $\phi_{(m+1)}:=0$.

The constraint equation (2.17) gives

$$
A_{j k}\left(\lambda_{j}-\lambda_{k}\right)=0=A_{k j}\left(\lambda_{k}-\lambda_{j}\right) .
$$

Here non-trivial solutions occur when $\lambda_{k}=\lambda_{j}$. This happens when $j, k$ belong to the same block $K_{(\ell)}$ and thus

$$
A=\sum_{\ell=0}^{m} A_{(\ell)}
$$


where

$$
A_{(\ell)}=\sum_{i \in K_{(\ell)}} A_{i} H_{i}+\sum_{\substack{j, k \in K_{(\ell)} \\ j<k}}\left(A_{j k} X_{e_{j}-e_{k}}+A_{k j} X_{e_{k}-e_{j}}\right) .
$$

This calculation also shows that the breaking of the original $\mathrm{U}(n)$ gauge symmetry to the centralizer subgroup (2.8) is given by

$$
\mathcal{H}=\prod_{\ell=0}^{m} \mathrm{U}\left(k_{\ell}\right)
$$

The $\mathfrak{u}(n)$-valued gauge potential $\mathcal{A}$ on $\mathcal{M}$ is by construction $\mathrm{SU}(2)$-invariant and decomposes into $k_{\ell} \times k_{\ell^{\prime}}$ blocks $\mathcal{A}^{\ell, \ell^{\prime}}$ with $\ell, \ell^{\prime}=0,1, \ldots, m$ and

$$
\begin{aligned}
\mathcal{A}^{\ell, \ell} & =A_{(\ell)}-\mathrm{a}_{(\ell)}, \\
\mathcal{A}^{\ell, \ell+1} & =-\phi_{(\ell+1)} \beta, \\
\mathcal{A}^{\ell+1, \ell} & =-\left(\mathcal{A}^{\ell, \ell+1}\right)^{\dagger}=\phi_{(\ell+1)}^{\dagger} \bar{\beta}, \\
\mathcal{A}^{\ell+i, \ell} & =0=\mathcal{A}^{\ell, \ell+i} \quad \text { for } \quad i \geq 2 .
\end{aligned}
$$

Here the local one-forms $\mathrm{a}_{(\ell)}$ on $\mathbb{C} P^{1}$ are given by

$$
\mathrm{a}_{(\ell)}=-\frac{\alpha_{\ell}(\bar{y} \mathrm{~d} y-y \mathrm{~d} \bar{y})}{1+y \bar{y}},
$$

and

$$
\beta=\frac{\mathrm{d} y}{1+y \bar{y}}, \quad \bar{\beta}=\frac{\mathrm{d} \bar{y}}{1+y \bar{y}}
$$

are the unique covariantly constant $\mathrm{SU}(2)$-invariant $(1,0)$ - and $(0,1)$-forms on $\mathbb{C} P^{1}$ respectively. From (3.17)-(3.20) it follows that the curvature two-form splits into $k_{\ell} \times k_{\ell^{\prime}}$ blocks

$$
\mathcal{F}^{\ell, \ell^{\prime}}=\mathrm{d} \mathcal{A}^{\ell, \ell^{\prime}}+\sum_{\ell^{\prime \prime}=0}^{m} \mathcal{A}^{\ell, \ell^{\prime \prime}} \wedge \mathcal{A}^{\ell^{\prime \prime}, \ell^{\prime}}
$$

and its only non-vanishing components are

$$
\begin{aligned}
\mathcal{F}^{\ell, \ell} & =F_{(\ell)}-\mathrm{f}_{(\ell)}+\left(\phi_{(\ell)}^{\dagger} \phi_{(\ell)}-\phi_{(\ell+1)} \phi_{(\ell+1)}^{\dagger}\right) \beta \wedge \bar{\beta}, \\
\mathcal{F}^{\ell, \ell+1} & =-\nabla \phi_{(\ell+1)} \wedge \beta \\
\mathcal{F}^{\ell+1, \ell} & =\nabla \phi_{(\ell+1)}^{\dagger} \wedge \bar{\beta}
\end{aligned}
$$

where

$$
\begin{aligned}
\mathrm{f}_{(\ell)} & =2 \alpha_{\ell} \beta \wedge \bar{\beta}, \\
F_{(\ell)} & =\mathrm{d} A_{(\ell)}+A_{(\ell)} \wedge A_{(\ell)}, \\
\nabla \phi_{(\ell+1)} & =\mathrm{d} \phi_{(\ell+1)}+A_{(\ell)} \phi_{(\ell+1)}-\phi_{(\ell+1)} A_{(\ell+1)}, \\
\nabla \phi_{(\ell+1)}^{\dagger} & =\mathrm{d} \phi_{(\ell+1)}^{\dagger}+A_{(\ell+1)} \phi_{(\ell+1)}^{\dagger}-\phi_{(\ell+1)}^{\dagger} A_{(\ell)}
\end{aligned}
$$

with $\phi_{(0)}:=0=: \phi_{(m+1)}$. 
The eigenvalues of the matrix $\Lambda$ from (3.6) are constrained by (2.12) to quantized values $\alpha_{\ell} \in \frac{1}{2} \mathbb{Z}$ given by

$$
\alpha_{\ell}=\frac{p+2 \ell}{2}
$$

for arbitrary $p \in \mathbb{Z}$. It follows that the matrix $\Lambda$ geometrically parameterizes the quantized magnetic charges of the unique $\mathrm{SU}(2)$-invariant family of monopole connections a $(\ell)$ on $\mathbb{C} P^{1}$. With $p=-m$ the Yang-Mills-Higgs model (2.34) reproduces the quiver gauge theories from [14] which are based on the linear $A_{m}$ quivers

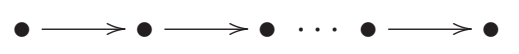

containing $m+1$ nodes corresponding to the gauge groups $\mathrm{U}\left(k_{\ell}\right)$ and gauge fields $A_{(\ell)}$, and $m$ arrows corresponding to the $\mathrm{U}\left(k_{\ell+1}\right) \times \mathrm{U}\left(k_{\ell}\right)$ bifundamental Higgs fields $\phi_{(\ell+1)}$. The quiver (3.27) characterizes how $\mathrm{SU}(2)$-invariance is incorporated into the gauge theory on $\mathcal{M}=M \times S^{2}$.

Note that this correspondence with quivers is somewhat symbolic, as an SU(2)equivariant principal $\mathcal{G}$-bundle does not belong to a representation category for the quiver (3.27). The association is possible because in the present case the gauge group $\mathcal{G}$ is a matrix Lie group: one may regard $\mathrm{U}\left(k_{\ell}\right)$ as the group of unitary automorphisms of a complex inner product space $V_{k_{\ell}} \simeq \mathbb{C}^{k_{\ell}}$ and the Higgs fields $\phi_{(\ell+1)}$ fibrewise as maps in $\operatorname{Hom}\left(V_{k_{\ell+1}}, V_{k_{\ell}}\right)$. To associate a quiver bundle to our construction we need a suitable representation of the quiver (3.27) in the category of vector bundles on $M$. For this, we can take the complex vector bundle $E=P \times_{\varrho} V$ on $\mathcal{M}$ associated to the fundamental representation $\varrho: \mathcal{G} \rightarrow \mathrm{U}(V)$ of $\mathcal{G}=\mathrm{U}(n)$ on $V \simeq \mathbb{C}^{n}$. Then the restriction $E_{M}:=\left.E\right|_{M \times\left[\mathbb{1}_{\mathrm{SU}(2)}\right]}=P_{M} \times\left._{\varrho} V\right|_{\mathcal{H}}$ is a $\mathrm{U}(1)$-equivariant vector bundle on $M$ with fibre the restriction $\left.V\right|_{\mathcal{H}}=\bigoplus_{\ell=0}^{m} V_{k_{\ell}}$ of the linear representation $(\varrho, V)$ to $\mathcal{H}$. The $\mathrm{U}(1)$-action on the fibre is given by $\left.\exp (t \Lambda)\right|_{V_{k_{\ell}}}=\mathrm{e}^{\mathrm{i} t\left(\frac{p}{2}+\ell\right)} \mathbb{1}_{V_{k_{\ell}}}$ and the Higgs fields are morphisms $\left.\Phi\right|_{E_{k_{\ell+1}}}: E_{k_{\ell+1}} \rightarrow E_{k_{\ell}}$ of the vector bundles $E_{k_{\ell}}:=P_{M} \times_{\varrho} V_{k_{\ell}}$ for each $\ell=0,1, \ldots, m$.

Our detailed treatment here of the standard case with $\mathcal{G}=\mathrm{U}(n)$ has the virtue that the exact same analysis can be performed for the remaining classical gauge groups $\mathcal{G}=\mathrm{SO}(2 n)$, $\mathrm{SO}(2 n+1)$, and $\mathrm{Sp}(2 n)$; the requisite group theory data for their decompositions in the Cartan-Weyl basis are summarised in appendix A. In every case one shows that, for generic eigenvalues $\alpha_{\ell}$ of the matrix $\Lambda$, the residual gauge symmetry group is again given by (3.16) (as a subgroup of $\mathcal{G}$ ) and the structure of the dimensionally reduced gauge theory can again be encoded in the $A_{m}$ quiver (3.27), with only trivial redefinitions of the coupling constants in (2.34) distinguishing the different cases. Such redefinitions may have implications in matching the quiver gauge theories with more realistic models as in [15].

\section{Covariant quiver gauge theories}

\subsection{Chern-Simons theory and transgression forms}

Let $P$ be a principal bundle with $(2 n+1)$-dimensional base space $\mathcal{M}$ and structure group $\mathcal{G}$. Let $\mathfrak{g}$ be the Lie algebra of $\mathcal{G}$ generated by $\mathrm{T}_{a}$ with $a=1, \ldots, \operatorname{dim} \mathfrak{g}$. Let $\mathcal{A}$ be a gauge 
potential on $\mathcal{M}$ defined as in (2.13) with

$$
\mathcal{A}=\mathcal{A}_{\mu^{\prime}} \mathrm{d} x^{\mu^{\prime}}=\mathcal{A}_{\mu^{\prime}}^{a} \mathrm{~d} x^{\mu^{\prime}} \otimes \mathrm{T}_{a}
$$

and let $\mathcal{F}$ be the curvature two-form (2.24). The product

$$
\chi^{(2 n+2)}(\mathcal{A})=\left\langle\mathcal{F}^{n+1}\right\rangle
$$

is a closed $(2 n+2)$-form on $\mathcal{M}$ which defines a characteristic class of $P$, where the bracket

$$
\langle-\rangle: \mathfrak{g}^{\otimes(n+1)} \longrightarrow \mathbb{R}
$$

denotes a symmetric $\mathfrak{g}$-invariant polynomial of rank $n+1$ which can always be determined once an explicit presentation of $\mathfrak{g}$ is specified. Since $\chi^{(2 n+2)}(\mathcal{A})$ is closed, it can be locally written as the exterior derivative of a Chern-Simons form

$$
\chi^{(2 n+2)}(\mathcal{A})=\mathrm{d} L_{\mathrm{CS}}^{(2 n+1)}
$$

where

$$
L_{\mathrm{CS}}^{(2 n+1)}=(n+1) \int_{0}^{1} \mathrm{~d} t\left\langle\mathcal{A} \wedge\left(t \mathrm{~d} \mathcal{A}+t^{2} \mathcal{A} \wedge \mathcal{A}\right)^{n}\right\rangle .
$$

The Chern-Simons form is, up to boundary terms, gauge-invariant. This means that under infinitesimal gauge transformations with parameter function $\lambda=\lambda^{a} \otimes \mathrm{T}_{a} \in \Omega^{0}(\mathcal{M}, \mathfrak{g})$, the variation of the gauge potential is given by

$$
\delta_{\lambda} \mathcal{A}=\mathrm{d} \lambda+[\mathcal{A}, \lambda]
$$

and subsequently (4.5) remains unchanged modulo boundary terms. Due to their quasigauge invariance property, Chern-Simons forms are good candidates to construct action functionals with the gauge potential $\mathcal{A}$ as the fundamental field, so we define

$$
S_{\mathrm{CS}}^{(2 n+1)}=\kappa(n+1) \int_{\mathcal{M}} \int_{0}^{1} \mathrm{~d} t\left\langle\mathcal{A} \wedge\left(t \mathrm{~d} \mathcal{A}+t^{2} \mathcal{A} \wedge \mathcal{A}\right)^{n}\right\rangle
$$

where $\kappa$ is a coupling constant.

Chern-Simons forms are a particular case of more general globally-defined differential forms. Consider the action functional

$$
S_{T}^{(2 n+1)}[\mathcal{A}, \overline{\mathcal{A}}]=\kappa \int_{\mathcal{M}} Q_{\mathcal{A} \leftarrow \overline{\mathcal{A}}}^{(2 n+1)}
$$

where $Q_{\mathcal{A} \leftarrow \overline{\mathcal{A}}}^{(2 n+1)}$ is the transgression form [18-21] defined by

$$
Q_{\mathcal{A} \leftarrow \overline{\mathcal{A}}}^{(2 n+1)}=-Q_{\overline{\mathcal{A}} \leftarrow \mathcal{A}}^{(2 n+1)}:=(n+1) \int_{0}^{1} \mathrm{~d} t\left\langle(\mathcal{A}-\overline{\mathcal{A}}) \wedge \mathcal{F}_{t}^{n}\right\rangle
$$

Here $\mathcal{A}$ and $\overline{\mathcal{A}}$ are two $\mathfrak{g}$-valued gauge potentials and we set

$$
\mathcal{A}_{t}=\overline{\mathcal{A}}+t(\mathcal{A}-\overline{\mathcal{A}}), \quad \mathcal{F}_{t}=\mathrm{d} \mathcal{A}_{t}+\mathcal{A}_{t} \wedge \mathcal{A}_{t}
$$


The action (4.8) is invariant under two different sets of symmetries. On the one hand, it is diffeomorphism invariant since it is constructed using only differential forms on $\mathcal{M}$, while on the other hand it possesses full invariance under local gauge transformations [22]

$$
\mathcal{A}^{g}=g^{-1} \mathcal{A} g+g^{-1} \mathrm{~d} g, \quad \overline{\mathcal{A}}^{g}=g^{-1} \overline{\mathcal{A}} g+g^{-1} \mathrm{~d} g
$$

where $g=\exp \left(\lambda^{a} \otimes \mathrm{T}_{a}\right) \in \Omega^{0}(\mathcal{M}, \mathcal{G})$. The Euler-Lagrange field equations associated to $(4.8)$ read as

$$
\left\langle\mathcal{F}^{n} \mathrm{~T}_{a}\right\rangle=0=\left\langle\overline{\mathcal{F}}^{n} \mathrm{~T}_{a}\right\rangle
$$

for $a=1, \ldots, \operatorname{dim} \mathfrak{g}$, subject to the boundary conditions

$$
\left.\int_{0}^{1} \mathrm{~d} t\left\langle\delta \mathcal{A}_{t} \wedge(\mathcal{A}-\overline{\mathcal{A}}) \wedge \mathcal{F}^{n-1}\right\rangle\right|_{\partial \mathcal{M}}=0
$$

for arbitrary variations $\delta \mathcal{A}_{t}$ of the gauge potentials. It is easy to check that the ChernSimons form is recovered in the limit $\overline{\mathcal{A}}=0$.

\subsection{Topological Chern-Simons-Higgs models}

We will now perform the SU(2)-equivariant dimensional reduction of the Chern-Simons gauge theory on $\mathcal{M}=M \times S^{2}$, where $M$ is an oriented manifold of dimension $d=2 n-1$. Throughout we assume that the manifold $M$ is closed, as no novel boundary effects arise in the models we derive. The gauge field defined by (2.14)-(2.16) can be written in the form

$$
\mathcal{A}=A-\mathrm{a}-\Phi \otimes \beta+\Phi^{\dagger} \otimes \bar{\beta},
$$

where

$$
\mathrm{a}:=\Lambda \otimes \frac{\mathrm{i}(\bar{y} \mathrm{~d} y-y \mathrm{~d} \bar{y})}{1+y \bar{y}}
$$

and we have used (3.22). In general, the computation of the reduced Chern-Simons action directly from its definition (4.7) is somewhat involved; to simplify the calculations considerably we use the subspace separation method [23] which provides a systematic way to compute Chern-Simons forms by making use of the extended Cartan homotopy formula [24] (see appendix B). This method has the virtue of separating the action into bulk and boundary contributions, and also splitting the Lagrangian into terms valued in a specified subspace decomposition of the gauge algebra.

The applicability of the method relies on regarding Chern-Simons forms as transgression forms that satisfy the triangle equation (see appendix B.3)

$$
Q_{\mathcal{A}_{2} \leftarrow \mathcal{A}_{0}}^{(2 n+1)}=Q_{\mathcal{A}_{2} \leftarrow \mathcal{A}_{1}}^{(2 n+1)}+Q_{\mathcal{A}_{1} \leftarrow \mathcal{A}_{0}}^{(2 n+1)}+\mathrm{d} Q_{\mathcal{A}_{2} \leftarrow \mathcal{A}_{1} \leftarrow \mathcal{A}_{0}}^{(2 n)},
$$

which decomposes a transgression form into the sum of two transgression forms depending on an intermediate connection plus an exact form with

$$
Q_{\mathcal{A}_{2} \leftarrow \mathcal{A}_{1} \leftarrow \mathcal{A}_{0}}^{(2 n)}:=n(n+1) \int_{0}^{1} \mathrm{~d} t \int_{0}^{t} \mathrm{~d} s\left\langle\left(\mathcal{A}_{2}-\mathcal{A}_{1}\right) \wedge\left(\mathcal{A}_{1}-\mathcal{A}_{0}\right) \wedge \mathcal{F}_{s, t}^{n-1}\right\rangle
$$

where $\mathcal{F}_{s, t}=\mathrm{d} \mathcal{A}_{s, t}+\mathcal{A}_{s, t} \wedge \mathcal{A}_{s, t}$ with $\mathcal{A}_{s, t}=s\left(\mathcal{A}_{2}-\mathcal{A}_{1}\right)+t\left(\mathcal{A}_{1}-\mathcal{A}_{0}\right)+\mathcal{A}_{0}$. The method then proceeds in three steps:

1. Decompose the gauge algebra $\mathfrak{g}$ into $p+1$ vector subspaces $\mathfrak{g}=\mathfrak{g}_{0} \oplus \mathfrak{g}_{1} \oplus \cdots \oplus \mathfrak{g}_{p}$. 
2. Expand the connections into components valued in each subspace as $\mathcal{A}=\boldsymbol{a}_{0}+\boldsymbol{a}_{1}+$ $\cdots+\boldsymbol{a}_{p}$ and $\overline{\mathcal{A}}=\overline{\boldsymbol{a}}_{0}+\overline{\boldsymbol{a}}_{1}+\cdots+\overline{\boldsymbol{a}}_{p}$ with $\boldsymbol{a}_{i}, \overline{\boldsymbol{a}}_{i} \in \mathfrak{g}_{i}$ for $i=0,1, \ldots, p$.

3. Evaluate the triangle equation (4.16) with the connections written in terms of pieces valued in each subspace as

$$
\mathcal{A}_{0}=\overline{\mathcal{A}}, \quad \mathcal{A}_{1}=\boldsymbol{a}_{0}+\boldsymbol{a}_{1}+\cdots+\boldsymbol{a}_{p-1}, \quad \mathcal{A}_{2}=\mathcal{A} .
$$

4. Repeat step 3 for the transgression form $Q_{\mathcal{A}_{1} \leftarrow \mathcal{A}_{0}}^{(2 n+1)}$, and so on.

For the present case we decompose $\mathfrak{g}=\mathfrak{g}_{0} \oplus \mathfrak{g}_{1}$ with $\mathfrak{g}_{0}=\mathfrak{h}$ and $\mathfrak{g}_{1}=\mathfrak{g} \ominus \mathfrak{h}$, and expand the gauge potential as

$$
\begin{aligned}
& \mathcal{A}_{0}=0, \\
& \mathcal{A}_{1}=-\mathrm{a}, \\
& \mathcal{A}_{2}=A-\mathrm{a}, \\
& \mathcal{A}_{3}=\Phi^{\dagger} \otimes \bar{\beta}-\Phi \otimes \beta+A-\mathrm{a} .
\end{aligned}
$$

By applying the triangle equation (4.16) we obtain the expression for the reduced ChernSimons action: the reduced Lagrangian splits into the sum of three terms

$$
\begin{aligned}
& L_{\Phi}=\kappa Q_{\mathcal{A}_{3} \leftarrow \mathcal{A}_{2}}^{(2 n+1)}=2 \kappa(n+1) \int_{0}^{1} \mathrm{~d} t\left\langle t\left(\Phi \nabla \Phi^{\dagger}-\Phi^{\dagger} \nabla \Phi\right) \wedge \beta \wedge \bar{\beta} \wedge F^{n-1}\right\rangle, \\
& L_{A}=\kappa Q_{\mathcal{A}_{2} \leftarrow \mathcal{A}_{1}}^{(2 n+1)}=2 \kappa(n+1) \int_{0}^{1} \mathrm{~d} t\left\langle 2 \mathrm{i} \Lambda \beta \wedge \bar{\beta} \wedge A \wedge\left(t \mathrm{~d} A+t^{2} A \wedge A\right)^{n-1}\right\rangle, \\
& L_{\Lambda}=\kappa Q_{\mathcal{A}_{1} \leftarrow \mathcal{A}_{0}}^{(2 n+1)}=0 .
\end{aligned}
$$

By integrating over $S^{2}$, the original $(2 n+1)$-dimensional Chern-Simons gauge theory reduces to a Chern-Simons-Higgs type model in $d=2 n-1$ dimensions with action

$$
S_{\mathrm{CSH}}^{(2 n-1)}=\kappa^{\prime} \int_{M} \int_{0}^{1} \mathrm{~d} t\left\langle t\left(\Phi \nabla \Phi^{\dagger}-\Phi^{\dagger} \nabla \Phi\right) \wedge F^{n-1}+2 \mathrm{i} \Lambda A \wedge\left(t \mathrm{~d} A+t^{2} A \wedge A\right)^{n-1}\right\rangle
$$

subject to the constraints $(2.17)-(2.19)$. Here we have defined $\kappa^{\prime}=8 \pi R^{2}(n+1) \kappa$ and the fields $F, \nabla \Phi$ are given by (2.30)-(2.31) respectively.

This action is "topological" in the sense that it is diffeomorphism invariant; this point is actually somewhat subtle and we return to it below. The first term of (4.24) is also manifestly invariant under the gauge transformations

$$
A^{h}=h^{-1} A h+h^{-1} \mathrm{~d} h, \quad \Phi^{h}=h^{-1} \Phi h
$$

for $h \in \Omega^{0}(M, \mathcal{H})$, but the second Chern-Simons type term is generically not: using [25, eq. (3.5)] one finds that the full action transforms as

$$
S_{\mathrm{CSH}}^{(2 n-1)}\left[A^{h}, \Phi^{h}\right]=S_{\mathrm{CSH}}^{(2 n-1)}[A, \Phi]-2 \mathrm{i}(-1)^{n} \frac{(n-1) ! n !}{(2 n-1) !} \kappa^{\prime} \int_{M}\left\langle\Lambda\left(h^{-1} \mathrm{~d} h\right)^{2 n-1}\right\rangle .
$$


Due to the constraint (2.12), the closed $(2 n-1)$-form $\left\langle\Lambda\left(h^{-1} \mathrm{~d} h\right)^{2 n-1}\right\rangle$ gives a de Rham representative for a class in the cohomology group $H^{2 n-1}\left(M, \pi_{2 n-1}(\mathcal{H})\right)$. Hence the deficit term in (4.26) generically vanishes if and only if the free part of the homotopy group $\pi_{2 n-1}(\mathcal{H})$ is trivial. Otherwise, the path integral for the quantum field theory is well-defined provided that the functional $\exp \left(\mathrm{i} S_{\mathrm{CSH}}^{(2 n-1)}\right)$ is invariant under gauge transformations; this requirement generically imposes a further topological quantization condition on the effective coupling constant $\kappa^{\prime}$ after dimensional reduction if the group $\pi_{2 n-1}(\mathcal{H}) / \operatorname{Tor}\left(\pi_{2 n-1}(\mathcal{H})\right)$ is non-trivial. Then up to a gauge transformation with parameter $\lambda=\xi\lrcorner A$, the infinitesimal action of diffeomorphisms of $M$ can be represented as contractions

$$
\left.\left.\delta_{\xi} A=\xi\right\lrcorner F, \quad \delta_{\xi} \Phi=\xi\right\lrcorner \nabla \Phi
$$

along vector fields $\xi \in \Omega^{0}(M, T M)$.

The field equations can be obtained by varying the reduced action (4.24) or equivalently by dimensional reduction over the general condition

$$
\delta S_{\mathrm{CS}}^{(2 n+1)}=\kappa \int_{\mathcal{M}}\left\langle\mathcal{F}^{n} \wedge \delta \mathcal{A}\right\rangle=0
$$

on $\mathcal{M}=M \times S^{2}$. One finds that the equations of motion reduce to

$$
\begin{aligned}
\left\langle\left(F^{n-1}\left(2 \mathrm{i} \Lambda-\left[\Phi, \Phi^{\dagger}\right]\right)+(n-1) F^{n-2} \wedge \nabla \Phi^{\dagger} \wedge \nabla \Phi\right) \wedge \delta A\right\rangle & =0, \\
& \left\langle F^{n-1} \wedge \nabla \Phi^{\dagger} \delta \Phi\right\rangle=0, \\
& \left\langle F^{n-1} \wedge \nabla \Phi \delta \Phi^{\dagger}\right\rangle=0,
\end{aligned}
$$

subject to the linear constraints (2.17)-(2.19). In the following we will study various aspects of the moduli space $\mathscr{M}_{n}$ of solutions to these equations modulo gauge transformations and diffeomorphisms. As a special class of topological solutions, note that the Higgs fields $\Phi$ are (locally) parallel sections of the adjoint bundle $\operatorname{ad}\left(P_{M}\right)$ if and only if the curvature two-form $F$ of $P_{M}$ vanishes, in which case the field equations are immediately satisfied when $n>1$. Since in this case the diffeomorphisms (4.27) vanish on-shell, this subspace of the solution space is the finite-dimensional moduli space of flat $\mathcal{H}$-connections on $M$ modulo gauge transformations, or equivalently the moduli space of representations of the fundamental group $\pi_{1}(M)$ in $\mathcal{H}$ modulo conjugation.

\subsection{Moduli spaces of solutions}

For some explicit examples, let us look at the case where $\mathcal{G}$ is one of the classical gauge groups from section 3 , focusing without loss of generality on $\mathcal{G}=\mathrm{U}(n)$. The dynamics of the reduced topological quiver gauge theory is then controlled by the invariant tensor associated to the residual gauge group (3.16). In general, if $\left\{\mathrm{t}_{a}\right\}_{a=1}^{\operatorname{dim} \mathfrak{h}}$ denotes the generators of the Lie algebra $\mathfrak{h}$ of $\mathcal{H}$, then the invariant tensor $g_{a_{1} \cdots a_{n+1}}$ is a symmetric tensor of rank $n+1$ that is invariant under the adjoint action of $\mathcal{H}$ which we take to be the symmetrized trace $[26]$

$$
g_{a_{1} \cdots a_{n+1}}=\left\langle\mathrm{t}_{a_{1}} \cdots \mathrm{t}_{a_{n+1}}\right\rangle=\frac{1}{(n+1) !} \sum_{\sigma \in S_{n+1}} \operatorname{Tr}\left(\mathrm{t}_{a_{\sigma(1)}} \cdots \mathrm{t}_{a_{\sigma(n+1)}}\right)
$$


where $S_{n+1}$ is the symmetric group of degree $n+1$. In the Cartan-Weyl basis the reduced gauge group $\mathcal{H}$ of (3.16) is generated by $\left\{H_{i}, X_{e_{j}-e_{k}}\right\}_{i, j, k=1}^{n}$. Let us now examine in detail some cases in lower dimensionalities.

$\boldsymbol{d}=1$. The non-zero components of the invariant tensor for $d=1$ coincide with the Killing-Cartan form

$$
\begin{aligned}
\left\langle X_{e_{j}-e_{k}} X_{e_{l}-e_{m}}\right\rangle & =\delta_{j m} \delta_{k l}, \\
\left\langle H_{i} X_{e_{j}-e_{k}}\right\rangle & =\delta_{i k} \delta_{i j}, \\
\left\langle H_{i} H_{j}\right\rangle & =\delta_{i j},
\end{aligned}
$$

and the resulting action functional is that of a topological matrix quantum mechanics given by

$$
S_{\mathrm{CSH}}^{(1)}=8 \pi R^{2} \kappa \int \mathrm{d} \tau \sum_{\ell=0}^{m} \operatorname{Tr}\left(\phi_{(\ell+1)} \nabla_{\tau} \phi_{(\ell+1)}^{\dagger}-\phi_{(\ell)}^{\dagger} \nabla_{\tau} \phi_{(\ell)}-2 \alpha_{\ell} A_{(\ell)}\right)
$$

where $\nabla_{\tau} \phi_{(\ell)}=\dot{\phi}_{(\ell)}+A_{(\ell-1)} \phi_{(\ell)}-\phi_{(\ell)} A_{(\ell)}$. In this case the gauge potentials $A_{(\ell)}(\tau) \in \mathfrak{h}$ are Lagrange multipliers and integrating them out of the action (4.32) yields the constraints

$$
\mu_{V}^{(\ell)}(\Phi):=\phi_{(\ell+1)} \phi_{(\ell+1)}^{\dagger}-\phi_{(\ell)}^{\dagger} \phi_{(\ell)}=2 \alpha_{\ell} \mathbb{1}_{k_{\ell}},
$$

while the remaining equations of motion for the Higgs fields read $\dot{\phi}_{(\ell)}=0=\dot{\phi}_{(\ell)}^{\dagger}$ for $\ell=0,1, \ldots, m$.

Thus in this case moduli space $\mathscr{M}_{1}$ of classical solutions is finite-dimensional and can be described as the subvariety cut out by the quadric (4.33) in the quotient of the affine variety $\prod_{\ell=0}^{m} \operatorname{Hom}\left(\mathbb{C}^{k_{\ell+1}}, \mathbb{C}^{k_{\ell}}\right)$ by the natural action of the gauge group (3.16) given by $\phi_{(\ell+1)} \mapsto g_{\ell+1} \phi_{(\ell+1)} g_{\ell}^{\dagger}$ with $g_{\ell} \in \mathrm{U}\left(k_{\ell}\right)$. The moduli space $\mathscr{M}_{1}$ also has a representation theoretic description as an affine quiver variety in the following way. The vector space of linear representations of the $A_{m}$ quiver (3.27) with fixed $\left.V\right|_{\mathcal{H}}=\bigoplus_{\ell=0}^{m} V_{k_{\ell}}$ is

$$
\mathscr{R}_{m}(V)=\bigoplus_{\ell=0}^{m} \operatorname{Hom}\left(V_{k_{\ell+1}}, V_{k_{\ell}}\right) .
$$

The corresponding representation space for the opposite quiver, obtained by reversing the directions of all arrows, is the dual vector space $\mathscr{R}_{m}(V)^{*}$ and the cotangent bundle on $\mathscr{R}_{m}(V)$ is

$$
T^{*} \mathscr{R}_{m}(V)=\mathscr{R}_{m}(V) \oplus \mathscr{R}_{m}(V)^{*} .
$$

It carries a canonical $\mathcal{H}$-invariant symplectic structure such that the linear $\mathcal{H}$-action on $T^{*} \mathscr{R}_{m}(V)$ is Hamiltonian [10] and the corresponding moment map is given by $\mu_{V}=$ $\left(\mu_{V}^{(\ell)}\right)_{\ell=0}^{m}: T^{*} \mathscr{R}_{m}(V) \rightarrow \mathfrak{h}^{*}$. The moduli space is then the symplectic quotient

$$
\mathscr{M}_{1}=\mu_{V}^{-1}\left(2 \alpha_{0}, 2 \alpha_{1}, \ldots, 2 \alpha_{m}\right) / / \mathcal{H} .
$$


This moduli space parameterizes isomorphism classes of semisimple representations of a certain preprojective algebra deformed by the eigenvalues $\alpha_{\ell}[10]$.

The topological nature of the quiver gauge theory in this instance is not surprising as the original pure three-dimensional Chern-Simons theory with Lagrangian

$$
L_{\mathrm{CS}}^{(3)}=\left\langle\mathcal{A} \wedge \mathrm{d} \mathcal{A}+\frac{1}{3} \mathcal{A} \wedge \mathcal{A} \wedge \mathcal{A}\right\rangle
$$

is a topological gauge theory, and hence so is its dimensional reduction. In this setting the affine quiver variety (4.36) is described geometrically as the finite-dimensional moduli space of flat $\mathrm{SU}(2)$-invariant $\mathcal{G}$-connections on the three-manifold $\mathcal{M}$, which can be regarded as the symplectic quotient of the space of all $\mathrm{SU}(2)$-invariant $\mathcal{G}$-connections on $\mathcal{M}$ by the action of the group of gauge transformations $\Omega^{0}(\mathcal{M}, \mathcal{H})$.

$\boldsymbol{d}=3$. The Chern-Simons-Higgs like system in the case $d=3$ is the three-dimensional diffeomorphism-invariant gauge theory reduced from pure $\mathrm{U}(n)$ Chern-Simons theory in five dimensions which has Lagrangian

$$
L_{\mathrm{CS}}^{(5)}=\left\langle\mathcal{A} \wedge(\mathrm{d} \mathcal{A})^{2}+\frac{3}{2} \mathcal{A}^{3} \wedge \mathrm{d} \mathcal{A}+\frac{3}{5} \mathcal{A}^{5}\right\rangle .
$$

As a consequence, the components of the invariant tensor are inherited from the fivedimensional theory and read as

$$
\begin{aligned}
\left\langle X_{e_{j}-e_{k}} X_{e_{j^{\prime}}-e_{k^{\prime}}} X_{e_{j^{\prime \prime}}-e_{k^{\prime \prime}}}\right\rangle & =\delta_{k j^{\prime}} \delta_{j k^{\prime \prime}} \delta_{k^{\prime} j^{\prime \prime}}+\delta_{k j^{\prime \prime}} \delta_{j k^{\prime}} \delta_{k^{\prime \prime} j^{\prime}} \\
\left\langle H_{j} X_{e_{j^{\prime}}-e_{k^{\prime}}} X_{e_{j^{\prime \prime}}-e_{k^{\prime \prime}}}\right\rangle & =\delta_{j j^{\prime}} \delta_{j k^{\prime \prime}} \delta_{k^{\prime} j^{\prime \prime}}+\delta_{j j^{\prime \prime}} \delta_{j k^{\prime}} \delta_{k^{\prime \prime} j^{\prime}} \\
\left\langle H_{j} H_{j^{\prime}} X_{e_{j^{\prime \prime}}-e_{k^{\prime \prime}}}\right\rangle & =\delta_{j j^{\prime}}\left(\delta_{j k^{\prime \prime}} \delta_{j^{\prime} j^{\prime \prime}}+\delta_{j j^{\prime \prime}} \delta_{k^{\prime \prime} j^{\prime}}\right) \\
\left\langle H_{j} H_{j^{\prime}} H_{j^{\prime \prime}}\right\rangle & =2 \delta_{j j^{\prime}} \delta_{j j^{\prime \prime}} \delta_{j^{\prime} j^{\prime \prime}}
\end{aligned}
$$

With this data, the reduced action becomes

$$
\begin{aligned}
S_{\mathrm{CSH}}^{(3)}=12 \pi R^{2} \kappa \int_{M} \sum_{\ell=0}^{m} \operatorname{Tr}( & \left(\phi_{(\ell+1)} \nabla \phi_{(\ell+1)}^{\dagger}-\phi_{(\ell)}^{\dagger} \nabla \phi_{(\ell)}\right) \wedge F_{(\ell)} \\
& \left.-2 \alpha_{\ell} A_{(\ell)} \wedge\left(\mathrm{d} A_{(\ell)}+\frac{2}{3} A_{(\ell)} \wedge A_{(\ell)}\right)\right)
\end{aligned}
$$

with the field equations

$$
\begin{aligned}
F_{(\ell)}\left(4 \alpha_{\ell}+\phi_{(\ell+1)} \phi_{(\ell+1)}^{\dagger}-\phi_{(\ell)}^{\dagger} \phi_{(\ell)}\right)-\nabla \phi_{(\ell)}^{\dagger} \wedge \nabla \phi_{(\ell)} & =0, \\
F_{(\ell)} \wedge \nabla \phi_{(\ell)}^{\dagger} & =0, \\
F_{(\ell)} \wedge \nabla \phi_{(\ell+1)} & =0 .
\end{aligned}
$$

Note that the pure gauge sector of this field theory is governed by the three-dimensional Chern-Simons action with gauge group $\mathcal{H}$, whose classical solution space is the moduli space of flat $\mathcal{H}$-connections on $M$ modulo gauge transformations. As an explicit example, 
consider the case $m=1$, so that the gauge group $\mathcal{G}=\mathrm{U}(2)$ is broken to $\mathcal{H}=\mathrm{U}(1) \times \mathrm{U}(1)$, and consider $A_{1}$ quiver gauge field configurations with $A_{(0)}=-A_{(1)}$ which further breaks the gauge symmetry to the diagonal $\mathrm{U}(1)$ subgroup of $\mathcal{H}$. It is then easy to reduce the field equations to the flatness conditions $F_{(0)}=-F_{(1)}=0$, and as a consequence there exists a local basis of parallel sections of the adjoint bundle $\operatorname{ad}\left(P_{M}\right)$. Hence in this case the solution space is again the finite-dimensional moduli space of flat $\mathcal{H}$-connections on $M$. Owing to the topological nature of the system in this dimensionality, we believe that this is the generic moduli space of solutions in this dimension, but we have no rigorous proof of this fact.

Reduced field equations similar to those of the $m=1$ case above were obtained in [16]. We note that one can consider regions of $M$ with monopole type Higgs field configurations having $\nabla \Phi=0$ but $F \neq 0$; in this case the monopole charge is non-zero only through twocycles of $M$ which enclose regions where $\nabla \Phi \neq 0$. According to the field equations (4.41), in such regions the Higgs fields must in addition satisfy $\left[\Phi, \Phi^{\dagger}\right]=2 \mathrm{i} \Lambda$, which is the minimum of the Higgs potential in (2.34). Thus monopole configurations are allowed in the Higgs vacuum and are triggered by spontaneous symmetry breaking. It would be interesting to examine the dynamics after symmetry breaking of the coupled Yang-Mills-Chern-SimonsHiggs models defined by the sum of the action functionals (2.34) and (4.24), along the lines of [15]; in this model the gauge sector also contains massive spin one degrees of freedom [27].

$\boldsymbol{d} \geq \mathbf{5}$. Although for $d=3$ the moduli space of solutions is completely classified by the topology of the manifold $M$ and hence has no local degrees of freedom, in dimensions $d \geq 5$ one can argue following [28-30] that the space of solutions of the diffeomorphism invariant Chern-Simons-Higgs model cannot be uniquely associated to the topology of $M$ as it generically contains local propagating degrees of freedom, depending on the algebraic properties of the invariant tensor. Our model presents an example of an irregular Hamiltonian system $[31,32]$ whose phase space is stratified into branches with different numbers of degrees of freedom and gauge symmetries, due to the dependence of the symplectic form on the fields. When certain generic conditions are fulfilled, the symplectic form is of maximal rank and it is shown by [29] using the standard Hamiltonian formalism that the number of local degrees of freedom in the pure gauge sector is given by

$$
\mathcal{N}=\frac{1}{2}(2(d-1) h-2(h+d-1)-(d-1)(h-1))=\frac{1}{2}(d-1)(h-1)-h,
$$

where $h>1$ is the dimension of the residual gauge group $\mathcal{H}$; the first term in (4.42) is the number of canonical variables, the second term is twice the number $h$ of first class constraints associated with the gauge symmetry plus $d-1$ first class constraints associated to spatial diffeomorphism invariance, and the third term corresponds to the second class constraints. Note that this number is zero only for $d=5$ and $h=2$, i.e. the $A_{1}$ quiver gauge theory in five dimensions with gauge group $\mathcal{H}=\mathrm{U}(1) \times \mathrm{U}(1)$.

There are also degenerate sectors where the rank of the symplectic form is smaller, additional local symmetries emerge, and fewer degrees of freedom propagate; on these branches the constraints are functionally dependent and the standard Dirac analysis is not applicable. Thus the dynamical structure of the theory changes throughout the phase 
space, from purely topological sectors to sectors with the maximal number (4.42) of local degrees of freedom. Moreover, the sector with maximal rank is stable under perturbations of the initial conditions, and on open neighbourhoods of the maximal rank solutions one can ignore the field-dependent nature of the constraints; on the contrary, degenerate sectors form measure zero subspaces of the phase space and around such degenerate backgrounds local degrees of freedom can propagate.

We do not think that this feature will be spoilt by the coupling to the Higgs fields, as the essential features should remain: the equations of motion do not constrain the connection to be flat. As our choice of invariant tensor (4.30) for $\mathcal{G}$ is primitive [26], we expect the generic condition to hold; note that this choice is the one that leads to the appropriate Higgs branching structure of the quiver gauge theory from section 3. In fact, the phase $F=0$ is degenerate because small perturbations around it are trivial. It would be interesting to see how the degree of freedom count (4.42) is modified by performing the analogous canonical analysis for the full Chern-Simons-Higgs model, but this seems far more complicated than the analysis of the pure Chern-Simons gauge theory. Moreover, even in the pure gauge sector, no explicit propagating solutions have been found thus far. If we choose to discard solutions with $F^{n}=0, n>1$ as degenerate backgrounds, then one can find a phase with $F$ of maximal rank which carries the maximum number of degrees of freedom (4.42). Such a propagating phase contains "Higgs monopole" type configurations analogous to those discussed above for the case $d=3$.

\section{Quiver gauge theory of AdS gravity}

\subsection{SU $(2,2 \mid 1)$ Chern-Simons supergravity}

The most general action for gravity in arbitrary dimensionality is given by the dimensional continuation of the Einstein-Hilbert action, called the Lovelock series [33-35]. In this expansion there are free parameters which cannot be fixed from first principles. However, in $D=2 n+1$ dimensions a special choice for the coefficients can be made in such a way that the Lovelock Lagrangian becomes a Chern-Simons form [36-39]. The importance of this feature lies in the fact that the gravity theory then possesses a gauge symmetry once the spin connection $\omega$ and the vielbein $e$ are arranged into a connection $\mathcal{A}$ valued in the Lie algebra of one of the Lie groups $\mathrm{SO}(D-1,2), \mathrm{SO}(D, 1)$ or $I \mathrm{SO}(D-1,1)$ corresponding respectively to the local isometry groups of spacetimes with negative, positive or vanishing cosmological constant. Another important reason for considering Chern-Simons gravity theories is that they admit natural supersymmetric extensions [25, 40, 41]. In this section we study as an example the $\mathrm{SU}(2)$-equivariant dimensional reduction of five-dimensional Chern-Simons supergravity on $\mathcal{M}=M \times S^{2}$, where $M$ is a three-manifold.

Five-dimensional supergravity can be constructed as a Chern-Simons gauge theory which is invariant under the supergroup $\mathrm{SU}(2,2 \mid N)$ [42]. The superalgebra $\mathfrak{s u}(2,2 \mid N)$ is the minimal supersymmetric extension of $\mathfrak{s u}(2,2)$, which is isomorphic to the anti-de Sitter (AdS) algebra $\mathfrak{s o}(4,2)$. A crucial observation is that in any dimension $D$ an explicit representation of the AdS algebra can be given in terms of gamma-matrices $\Gamma_{a}$ which satisfy 
the Clifford algebra relations (see appendix C)

$$
\left\{\Gamma_{a}, \Gamma_{b}\right\}=2 \eta_{a b}
$$

where $\eta=\operatorname{diag}(-1,1, \ldots, 1)$ is the metric of $D$-dimensional Minkowski space. By defining

$$
\Gamma_{a b}=\frac{1}{2}\left[\Gamma_{a}, \Gamma_{b}\right]
$$

it is easy to show that

$$
\begin{aligned}
{\left[\Gamma_{a}, \Gamma_{b}\right] } & =2 \Gamma_{a b} \\
{\left[\Gamma_{a b}, \Gamma_{c d}\right] } & =2\left(\eta_{c b} \Gamma_{a b}-\eta_{c a} \Gamma_{b d}+\eta_{d b} \Gamma_{c a}-\eta_{d a} \Gamma_{c b}\right) \\
{\left[\Gamma_{a b}, \Gamma_{c}\right] } & =2\left(\eta_{c b} \Gamma_{a}-\eta_{c a} \Gamma_{b}\right)
\end{aligned}
$$

In this way, by choosing a set of $4 \times 4$ matrices satisfying (5.3)-(5.5) it is possible to represent the Lie algebra $\mathfrak{s u}(2,2)$ as a matrix algebra by defining

$$
\mathrm{J}_{a b}=\frac{1}{2} \Gamma_{a b}, \quad \mathrm{P}_{a}=\frac{1}{2} \Gamma_{a} .
$$

Let us now turn to the supersymmetric extension $\mathfrak{s u}(2,2 \mid N)$. For definiteness, we consider the case $N=1$ which accommodates the minimum number $\mathcal{N}=2$ of supersymmetries. A representation of $\mathfrak{s u}(2,2 \mid 1)$ can be obtained by extending the bosonic generators $\left\{\mathrm{P}_{a}, \mathrm{~J}_{a b}\right\}$ as

$$
\mathrm{P}_{a}=\left(\begin{array}{cc}
\frac{1}{2}\left(\Gamma_{a}\right)_{\beta}^{\alpha} & 0 \\
0 & 0
\end{array}\right), \quad \mathrm{J}_{a b}=\left(\begin{array}{cc}
\frac{1}{2}\left(\Gamma_{a b}\right)_{\beta}^{\alpha} & 0 \\
0 & 0
\end{array}\right)
$$

and inserting the fermionic generators

$$
\mathrm{Q}^{\gamma}=\left(\begin{array}{cc}
0 & 0 \\
-2 \delta_{\beta}^{\gamma} & 0
\end{array}\right), \quad \overline{\mathbf{Q}}_{\gamma}=\left(\begin{array}{cc}
0 & -2 \delta_{\gamma}^{\alpha} \\
0 & 0
\end{array}\right) .
$$

The supersymmetry algebra further requires the inclusion of a $U(1)$ generator

$$
\mathrm{K}=\left(\begin{array}{cc}
\frac{\mathrm{i}}{4} \delta^{\alpha} & 0 \\
0 & \mathrm{i}
\end{array}\right)
$$

so that gauge invariance is preserved [43].

\subsection{Dimensional reduction}

In order to perform the $\mathrm{SU}(2)$-equivariant dimensional reduction of $\mathrm{SU}(2,2 \mid 1)$ ChernSimons supergravity, we choose the element $\Lambda$ to take values in the Lorentz subalgebra $\mathfrak{s o}(1,4)$ generated by $\left\{J_{a b}\right\}$ and expand it as

$$
\Lambda=\frac{\mathrm{i}}{2} \lambda^{a b} \mathrm{~J}_{a b} .
$$


This choice is not arbitrary, in the sense that it is the only one that leads to an EinsteinHilbert term after dimensional reduction. Furthermore, non-trivial solutions of the constraint equations (2.18)-(2.19) are possible only if the Higgs fields $\Phi$ take values in the fermionic sector of $\mathfrak{s u}(2,2 \mid 1)$; we expand them as

$$
\Phi=\bar{Q}_{\beta} \chi^{\beta}, \quad \bar{\Phi}=\bar{\chi}_{\beta} Q^{\beta}
$$

where $\chi$ and $\bar{\chi}$ are four-component Dirac spinor zero-forms with $\beta$ running over $1,2,3,4$. In this way the constraints $(2.18)-(2.19)$ read as

$$
\left(\frac{1}{4} \lambda^{a b}\left(\Gamma_{a b}\right)_{\beta}^{\alpha}+\delta_{\beta}^{\alpha}\right) \chi^{\beta}=0, \quad \bar{\chi}_{\alpha}\left(\frac{1}{4} \lambda^{a b}\left(\Gamma_{a b}\right)_{\beta}^{\alpha}+\delta_{\beta}^{\alpha}\right)=0 .
$$

Gauging the Lie superalgebra $\mathfrak{s u}(2,2 \mid 1)$ means that the gauge potential decomposes as

$$
A=\frac{1}{2} \omega^{a b} \mathrm{~J}_{a b}+e^{a} \mathrm{P}_{a}+b \mathrm{~K}+\bar{\psi}_{\alpha} \mathrm{Q}^{\alpha}-\overline{\mathrm{Q}}_{\beta} \psi^{\beta}
$$

where $e, \omega$ are the standard vielbein and spin connection, $b$ is a $\mathrm{U}(1)$ gauge field and $\psi, \bar{\psi}$ are four-component spin $\frac{3}{2}$ gravitino fields. The constraint equation (2.17) reads

$$
\begin{aligned}
\lambda_{b}^{a} \omega^{b d} & =0, \\
\lambda_{b}^{a} e^{b} & =0, \\
\bar{\psi}_{\alpha} \lambda^{a b}\left(\Gamma_{a b}\right)_{\beta}^{\alpha} & =0, \\
\lambda^{a b}\left(\Gamma_{a b}\right)_{\beta}^{\alpha} \psi^{\beta} & =0 .
\end{aligned}
$$

These equations are still generic and will characterize the symmetry breaking pattern once the non-zero components of $\lambda^{a b}$ are specified. For this, we choose a particular representation of $\mathfrak{s u}(2,2 \mid 1)$. Using the Pauli matrices (2.9), a representation of the Clifford algebra in five dimensions is given by

$$
\begin{aligned}
& \Gamma_{0}=\mathrm{i} \sigma_{1} \otimes \mathbb{1}_{2}, \\
& \Gamma_{1}=\sigma_{2} \otimes \mathbb{1}_{2}, \\
& \Gamma_{2}=\sigma_{3} \otimes \sigma_{1}, \\
& \Gamma_{3}=\sigma_{3} \otimes \sigma_{2}, \\
& \Gamma_{4}=\sigma_{3} \otimes \sigma_{3} .
\end{aligned}
$$

The explicit construction is detailed in appendix C.2. We now restrict $\lambda^{a b} \mathrm{~J}_{a b}$ to be $\lambda^{01} \mathrm{~J}_{01}$; other restrictions are possible and they all lead to the same qualitative results below. With this choice the algebraic quantization condition (2.12) is satisfied and the constraint equation (5.12) has non-trivial solutions if $\lambda^{01}=4$. In that case, one finds

$$
\chi^{2}=\chi^{4}=0=\bar{\chi}^{2}=\bar{\chi}^{4} .
$$


Similarly, non-trivial solutions of (5.14)-(5.17) are given by taking

$$
\omega^{1 a}=0=\omega^{0 a}, \quad e^{1}=0=e^{0}, \quad \bar{\psi}_{\alpha}=0=\psi^{\alpha}
$$

for $a=0,1,2,3,4$ and $\alpha=1,2,3,4$.

The reduced field content can therefore be summarised as

$$
\begin{aligned}
& e^{a}, \omega^{a b} \quad \text { for } \quad a, b=2,3,4, \\
& \chi_{\alpha}, \bar{\chi}^{\alpha} \quad \text { for } \quad \alpha=1,3, \\
& b \quad \text { as } \mathrm{U}(1) \text { gauge field. }
\end{aligned}
$$

Since the reduced gauge potential becomes

$$
A=\frac{1}{2} \omega^{a b} \mathrm{~J}_{a b}+e^{a} \mathrm{P}_{a}+b \mathrm{~K} \in \mathfrak{s o}(2,2) \oplus \mathfrak{u}(1),
$$

the gauge symmetry $\mathcal{G}=\mathrm{SU}(2,2 \mid 1)$ is broken by this construction to

$$
\mathcal{H}=\mathrm{SO}(2,2) \times \mathrm{U}(1) .
$$

The quiver gauge theory is thus based on the $A_{1}$ quiver

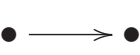

with the left node containing the $\mathrm{SO}(2,2)$ gravitational content $e, \omega$, the right node containing the $\mathrm{U}(1)$ gauge field $b$, and the arrow corresponding to the Higgs fermions $\chi$ and $\bar{\chi}$ which transform in the bifundamental representation of $\mathrm{SO}(2,2) \times \mathrm{U}(1)$. Since $\pi_{3}(\mathrm{U}(1))=0=\pi_{3}(\mathrm{SO}(2,2))$, there is no topological quantization condition required of the gravitational constant $\kappa^{\prime}$ after dimensional reduction.

In order to evaluate the reduced Chern-Simons-Higgs action, note that the curvature two-form associated to the group $\mathrm{SO}(2,2) \times \mathrm{U}(1)$ is

$$
F=\frac{1}{2}\left(R^{a b}+\frac{1}{l^{2}} e^{a} \wedge e^{b}\right) \mathrm{J}_{a b}+\frac{1}{l} T^{a} \mathrm{P}_{a}+\mathrm{d} b \mathrm{~K}
$$

where $l$ is the AdS radius, $R^{a b}=\mathrm{d} \omega^{a b}+\omega^{a}{ }_{c} \wedge \omega^{c b}$ is the Lorentz curvature two-form, and $T^{a}=\mathrm{d} e^{a}+\omega_{b}^{a} \wedge e^{b}$ is the torsion two-form. The non-vanishing components of the $\mathfrak{s u}(2,2 \mid 1)$-invariant tensor of rank three are given in appendix C.2. With this, one finds that the Chern-Simons-Higgs gravitational action is given by

$$
S_{\mathrm{CSH}}^{(3)}=\frac{\kappa^{\prime}}{l} \int_{M}\left(\epsilon_{a b c}\left(R^{a b}+\frac{1}{3 l^{2}} e^{a} \wedge e^{b}\right) \wedge e^{c}-\mathrm{i} \nabla \bar{\chi}_{\alpha} \wedge \mathcal{Z}_{\beta}^{\alpha} \chi^{\beta}+\mathrm{i} \bar{\chi}_{\alpha} \mathcal{Z}_{\beta}^{\alpha} \wedge \nabla \chi^{\beta}\right)
$$

where $\kappa^{\prime}=8 \pi R^{2} \kappa$ and

$$
\begin{aligned}
\mathcal{Z}_{\beta}^{\alpha} & =\frac{1}{2}\left(R^{a b}+\frac{1}{l^{2}} e^{a} \wedge e^{b}\right)\left(\Gamma_{a b}\right)_{\beta}^{\alpha}-\frac{1}{l} T^{a}\left(\Gamma_{a}\right)_{\beta}^{\alpha}+\frac{5 \mathrm{i}}{2} \delta_{\beta}^{\alpha} \mathrm{d} b, \\
\nabla \bar{\chi}_{\alpha} & =\mathrm{d} \bar{\chi}_{\alpha}-\frac{1}{4} \bar{\chi}_{\alpha} \omega^{a b}\left(\Gamma_{a b}\right)_{\beta}^{\alpha}-\frac{1}{2} \bar{\chi}_{\alpha} e^{a}\left(\Gamma_{a}\right)_{\beta}^{\alpha}+\frac{3 \mathrm{i}}{4} b \bar{\chi}_{\alpha} \delta_{\beta}^{\alpha}, \\
\nabla \chi^{\beta} & =\mathrm{d} \chi^{\beta}+\frac{1}{4} \omega^{a b}\left(\Gamma_{a b}\right)_{\beta}^{\alpha} \chi^{\beta}+\frac{1}{2} e^{a}\left(\Gamma_{a}\right)_{\beta}^{\alpha} \chi^{\beta}-\frac{3 \mathrm{i}}{4} b \delta_{\beta}^{\alpha} \chi^{\beta} .
\end{aligned}
$$


Note that the reduced field content restricts the gamma-matrices of the five-dimensional representation according to

$$
\begin{aligned}
\Gamma_{0}=\left(\begin{array}{cc}
0 & \mathrm{i} \\
\mathrm{i} & 0
\end{array}\right), & \Gamma_{1}=\left(\begin{array}{cc}
0 & -\mathrm{i} \\
\mathrm{i} & 0
\end{array}\right), & \Gamma_{2}=\left(\begin{array}{cc}
1 & 0 \\
0 & -1
\end{array}\right), \\
\Gamma_{01}=\left(\begin{array}{cc}
-1 & 0 \\
0 & 1
\end{array}\right), & \Gamma_{02}=\left(\begin{array}{cc}
0 & -\mathrm{i} \\
\mathrm{i} & 0
\end{array}\right), & \Gamma_{12}=\left(\begin{array}{ll}
0 & \mathrm{i} \\
\mathrm{i} & 0
\end{array}\right),
\end{aligned}
$$

which gives a representation of the Clifford algebra in $d=2+1$ dimensions.

The infinitesimal gauge transformations corresponding to (4.25) yield local symmetry transformations for the gauge fields and Higgs fermions given by

$$
\begin{aligned}
\delta_{\lambda, \rho} \omega^{a b} & =\mathrm{d} \lambda^{a b}+\omega_{c}^{a} \lambda^{c b}+\omega_{c}^{b} \lambda^{a c}+\frac{1}{l^{2}}\left(e^{a} \wedge \rho^{b}-\rho^{a} \wedge e^{b}\right), \\
\delta_{\lambda, \rho} e^{a} & =\mathrm{d} \rho^{a}+\omega^{a}{ }_{b} \rho^{b}-\lambda_{b}^{a} e^{b}, \\
\delta_{\beta} b & =\mathrm{d} \beta \\
\delta_{\rho, \kappa, \beta} \chi & =\frac{1}{2 l} \rho^{a} \Gamma_{a} \chi-\frac{1}{2} \epsilon_{a b c} \kappa^{a b} \Gamma^{c} \chi-\frac{3 \mathrm{i}}{4} \beta \chi, \\
\delta_{\rho, \kappa, \beta} \bar{\chi} & =-\frac{1}{2 l} \bar{\chi} \rho^{a} \Gamma_{a}+\frac{1}{2} \epsilon_{a b c} \bar{\chi} \kappa^{a b} \Gamma^{c}+\frac{3 \mathrm{i}}{4} \bar{\chi} \beta .
\end{aligned}
$$

The action (5.30) describes a theory of Einstein-Hilbert gravity with cosmological constant in three dimensions, plus a non-minimal coupling between Higgs fermions and the fields associated to the curvature of the residual gauge symmetry $\mathrm{SO}(2,2) \times \mathrm{U}(1)$. This model is not supersymmetric as one sees from the gauge transformations (5.36)-(5.37). The equivariant dimensional reduction scheme thus provides a novel and systematic way to couple scalar fermions to gravitational theories, which is normally cumbersome to do.

The variation of the Chern-Simons-Higgs action (5.30) leads to the field equations

$$
\begin{aligned}
2 \epsilon_{a b c} \check{R}^{a b}+\frac{\mathrm{i}}{l} T_{c} \bar{\chi} \chi-\frac{1}{2} \mathrm{~d} b \bar{\chi} \Gamma_{c} \chi-\mathrm{i} \nabla \bar{\chi} & \wedge \Gamma_{c} \nabla \chi \\
\mathrm{i} \check{R}^{a b} \bar{\chi} \chi+\frac{1}{l} \epsilon^{a b c} T_{c}+\frac{1}{4} \mathrm{~d} b \bar{\chi} \Gamma^{a b} \chi-\mathrm{i} \nabla \bar{\chi} \wedge \Gamma^{a b} \nabla \chi & =0, \\
\check{R}^{a b} \bar{\chi} \Gamma_{a b} \chi-\frac{1}{l} T^{a} \bar{\chi} \Gamma_{a} \chi+\frac{15 \mathrm{i}}{2} \mathrm{~d} b \bar{\chi} \chi & =0, \\
\mathcal{Z} \wedge \nabla \chi & =0, \\
\nabla \bar{\chi} & \wedge \mathcal{Z}=0,
\end{aligned}
$$

where we have used the abbreviation

$$
\check{R}^{a b}:=\frac{1}{2}\left(R^{a b}+\frac{1}{l^{2}} e^{a} \wedge e^{b}\right) .
$$

These equations demonstrate an interesting coupling between curvature and the matter currents; note that at least one of the torsion field $T^{a}$ or the $\mathrm{U}(1)$ field strength $\mathrm{d} b$ must 
be non-zero to get a non-trivial matter coupling; otherwise, when $T^{a}=0=\mathrm{d} b$ the matter fields freely decouple from gravity and the field equations reduce to those of pure AdS gravity in three dimensions.

\subsection{Applications}

We close with some brief discussion about possible generalizations and applications of the gravity theories described in this section. Five-dimensional supergravity serves as an interesting testing ground for string theory; its Lagrangian can be obtained via dimensional reduction of 11-dimensional supergravity where it inherits the Chern-Simons term for the $\mathrm{U}(1)$ gauge field $b$ from reduction of the four-form term. Non-trivial stationary solutions of the matter-coupled gravity theory (5.30) on $M$ can lead to non-asymptotically flat stationary solutions of the original five-dimensional supergravity theory on $\mathcal{M}=M \times S^{2}$. In particular, it would be interesting to seek BTZ-type solutions of the field equations (5.38). Note that by restricting to the bosonic sector by setting all fermions to zero, our reduction reduces ordinary five-dimensional AdS gravity to three-dimensional AdS gravity without any matter fields; hence our reduction scheme further provides a means for lifting purely gravitational configurations on $M$ to solutions on $M \times S^{2}$, and it would be interesting to examine this lifting in more detail on some explicit solutions.

The extension of this analysis to supergroups $\mathrm{SU}(2,2 \mid N)$ with $N>1$ would lead to a quiver gauge theory based on the $A_{1}$ quiver with residual gauge group $\mathcal{H}=\mathrm{SO}(2,2) \times \mathrm{U}(N)$, along with additional $\mathrm{SU}(N)$ gauge fields and gravitinos (see appendix C.1). The extensions to higher dimensions could presumably also lead to novel quiver gauge theories of gravitymatter interactions. Our construction here is similar to the known method of compactifying Einstein-Maxwell theories over $S^{2}$ supported by magnetic monopole flux (see e.g. [44]); this technique can be used to export non-vacuum solutions with isometry group $\mathrm{SO}(2,2) \times \mathrm{SO}(3)$ to local $A d S_{3} \times S^{2}$ solutions of the five-dimensional Einstein equations, which in the minimal supergravity case are near-horizon limits of black strings.

It would be interesting to extend the present construction to a quiver gauge theory of higher-spin gravity in three dimensions, which requires extending the three-dimensional Chern-Simons gauge theory based on the AdS group $\mathrm{SO}(2,2) \simeq \mathrm{SL}(2, \mathbb{R}) \times \mathrm{SL}(2, \mathbb{R})$ to those based on non-compact real forms of $\operatorname{SL}(n, \mathbb{C}) \times \operatorname{SL}(n, \mathbb{C})$ for $n>2$ (see e.g. [45]). Finding the appropriate Chern-Simons supergravity theory in higher dimensions could then be used as a novel mechanism to couple matter fields to higher-spin gravity theories. Moreover, by assigning different coupling constants to the two $\mathrm{SL}(2, \mathbb{R})$ factors, one can couple fermionic matter fields to the Chern-Simons gauge theory of gravity on three-dimensional RiemannCartan spacetimes considered in e.g. [46].

\section{Acknowledgments}

We thank L. Griguolo, F. Izaurieta, O. Miskovic, E. Rodriguez, P. Salgado and D. Seminara for helpful discussions and correspondence. This work was supported in part by the Consolidated Grant ST/J000310/1 from the U.K. Science and Technology Facilities Coun- 
cil. The work of O.V. is supported by grants from the Comisión Nacional de Investigación Científica y Tecnológica CONICYT and from the Universidad de Concepción, Chile.

\section{A Classical gauge groups}

In this appendix we summarize the group theory data which are used in section 3 in the case when the gauge symmetry belongs to one of the four infinite families $A_{n}, B_{n}, C_{n}, D_{n}$ of classical Lie groups in the Cartan classification; we consider each family in turn. Below $\left\{E_{i, j}\right\}_{i, j=1}^{n}$ denotes the orthonormal basis of $n \times n$ matrix units with elements $\left(E_{i, j}\right)_{k l}=$ $\delta_{i k} \delta_{j l}$, and $\left\{e_{i}\right\}_{i=1}^{n}$ is the canonical orthonormal basis of $\mathbb{R}^{n}$.

$\mathcal{G}=\mathrm{U}(n)$

\begin{tabular}{|l|l|l|}
\hline Positive roots $\alpha>0$ & $e_{i}-e_{j}$ & $1 \leq i<j \leq n$ \\
\hline Cartan generators & $H_{i}=E_{i, i}$ & $1 \leq i \leq n$ \\
\hline Root vectors & $X_{e_{i}-e_{j}}=E_{i, j}$ & $i \neq j, i, j=1, \ldots, n$ \\
\hline Weyl symmetry $\mathcal{W}$ & $S_{n}$ & \\
\hline
\end{tabular}

$\mathcal{G}=\operatorname{SO}(2 n+1)$

\begin{tabular}{|l|l|l|}
\hline Positive roots $\alpha>0$ & $e_{i} \pm e_{j}$ & $1 \leq i<j \leq n$ \\
& $e_{i}$ & $1 \leq i \leq n$ \\
\hline Cartan generators & $H_{i}=E_{i, i}-E_{i+n, i+n}$ & $1 \leq i \leq n$ \\
\hline Root vectors & $X_{e_{i}-e_{j}}=E_{j+1, i+1}-E_{i+n+1, j+n+1}$ & $i \neq j$ \\
& $X_{e_{i}+e_{j}}=E_{i+n+1, j+1}-E_{j+n+1, i+1}$ & $i<j$ \\
& $X_{e_{i}}=E_{1, i+1}-E_{i+n+1,1}$ & $1 \leq i \leq n$ \\
\hline Weyl symmetry $\mathcal{W}$ & $S_{n} \ltimes\left(\mathbb{Z}_{2}\right)^{n}$ & \\
\hline
\end{tabular}

$\mathcal{G}=\operatorname{Sp}(2 n)$

\begin{tabular}{|l|l|l|}
\hline Positive roots $\alpha>0$ & $e_{i} \pm e_{j}$ & $1 \leq i<j \leq n$ \\
& $e_{2 i}$ & $1 \leq i \leq n$ \\
\hline Cartan generators & $H_{i}=E_{i, i}-E_{i+n, i+n}$ & $1 \leq i \leq n$ \\
\hline Root vectors & $X_{e_{i}-e_{j}}=E_{j, i}-E_{i+n, j+n}$ & $i \neq j$ \\
& $X_{e_{i}+e_{j}}=E_{i+n, j}-E_{j+n, i}$ & $i<j$ \\
& $X_{2 e_{i}}=E_{i+n, i}$ & $1 \leq i \leq n$ \\
\hline Weyl symmetry $\mathcal{W}$ & $S_{n} \ltimes\left(\mathbb{Z}_{2}\right)^{n}$ & \\
\hline
\end{tabular}

$\mathcal{G}=\mathrm{SO}(2 n)$

\begin{tabular}{|l|l|l|}
\hline Positive roots $\alpha>0$ & $e_{i} \pm e_{j}$ & $1 \leq i<j \leq n$ \\
\hline Cartan generators & $H_{i}=E_{i, i}-E_{i+n, i+n}$ & $1 \leq i \leq n$ \\
\hline Root vectors & $X_{e_{i}-e_{j}}=E_{j, i}-E_{i+n, j+n}$ & $i \neq j$ \\
& $X_{e_{i}+e_{j}}=E_{i+n, j}-E_{j+n, i}$ & $i<j$ \\
& $X_{-e_{i}-e_{j}}=E_{j, i+n}-E_{i, j+n}$ & $i<j$ \\
\hline Weyl symmetry $\mathcal{W}$ & $S_{n} \ltimes\left(\mathbb{Z}_{2}\right)^{n-1}$ & \\
\hline
\end{tabular}




\section{B Extended Cartan homotopy formula}

\section{B.1 Restricted homotopy formula}

Fix $r \geq 0$ and consider a set of gauge connection one-forms $\mathcal{A}_{i}$ with $i=0,1, \ldots, r+1$ on a $D$-dimensional manifold $\mathcal{M}$, together with a Euclidean $(r+1)$-simplex $\Delta_{r+1} \subset \mathbb{R}^{r+2}$ defined by

$$
\Delta_{r+1}:=\left\{t=\left(t_{i}\right)_{i=0}^{r+1} \mid t_{i} \geq 0, \sum_{i=0}^{r+1} t_{i}=1\right\} .
$$

We will sometimes write this as $\Delta_{r+1}=\left\langle t_{0}, t_{1}, \ldots, t_{r+1}\right\rangle$. For $t \in \Delta_{r+1}$ the linear combination

$$
\mathcal{A}_{t}=\sum_{i=0}^{r+1} t_{i} \mathcal{A}_{i}
$$

transforms as a gauge connection in the same way as any individual form $\mathcal{A}_{i}$. Its curvature two-form is $\mathcal{F}_{t}=\mathrm{d} \mathcal{A}_{t}+\mathcal{A}_{t} \wedge \mathcal{A}_{t}$. Then the extended Cartan homotopy formula is given by

$$
\int_{\partial \Delta_{r+1}} \frac{\mathrm{h}_{t}^{p}}{p !} \Pi=\int_{\Delta_{r+1}} \frac{\mathrm{h}_{t}^{p+1}}{(p+1) !} \mathrm{d} \Pi+(-1)^{p+q} \mathrm{~d} \int_{\Delta_{r+1}} \frac{\mathrm{h}_{t}^{p+1}}{(p+1) !} \Pi
$$

where generally $\Pi$ is a polynomial in the fields $\left\{\mathcal{A}_{t}, \mathcal{F}_{t}, \mathrm{~d}_{t} \mathcal{A}_{t}, \mathrm{~d}_{t} \mathcal{F}_{t}\right\}$ which is simultaneously an $m$-form on $\mathcal{M}$ and a $q$-form on $\Delta_{r+1}$ with $m \geq p$ and $p+q=r$; here we denote by $\mathrm{d}_{t}$ the exterior derivative on $\Delta_{r+1}$. The operator $\mathrm{h}_{t}$ is the homotopy derivation which maps differential forms on $\mathcal{M} \times \Delta_{r+1}$ according to

$$
\mathrm{h}_{t}: \Omega^{a}(\mathcal{M}) \otimes \Omega^{b}\left(\Delta_{r+1}\right) \longrightarrow \Omega^{a-1}(\mathcal{M}) \otimes \Omega^{b+1}\left(\Delta_{r+1}\right)
$$

and satisfies the Leibniz rule. The action of $\mathrm{h}_{t}$ on $\mathcal{A}_{t}, \mathcal{F}_{t}$ is given by

$$
\mathrm{h}_{t} \mathcal{F}_{t}=\mathrm{d}_{t} \mathcal{A}_{t}, \quad \mathrm{~h}_{t} \mathcal{A}_{t}=0
$$

The operators $\mathrm{h}_{t}, \mathrm{~d}_{t}$ and $\mathrm{d}$ define a graded algebra

$$
\mathrm{d}^{2}=0=\mathrm{d}_{t}^{2}, \quad\left[\mathrm{~h}_{t}, \mathrm{~d}\right]=\mathrm{d}_{t}, \quad\left\{\mathrm{~d}, \mathrm{~d}_{t}\right\}=0=\left[\mathrm{h}_{t}, \mathrm{~d}_{t}\right] .
$$

Let us look now at the particular choice of polynomial

$$
\Pi=\left\langle\mathcal{F}_{t}^{n+1}\right\rangle .
$$

For this choice $\mathrm{d} \Pi=0, q=0$ and $m=2 n+2$, so that the allowed values for $p$ are $p=0,1, \ldots, 2 n+2$. In this case the homotopy formula reduces to

$$
\int_{\partial \Delta_{p+1}} \frac{\mathrm{h}_{t}^{p}}{p !}\left\langle\mathcal{F}_{t}^{n+1}\right\rangle=(-1)^{p} \mathrm{~d} \int_{\Delta_{p+1}} \frac{\mathrm{h}_{t}^{p+1}}{(p+1) !}\left\langle\mathcal{F}_{t}^{n+1}\right\rangle,
$$

which is known as the restricted or closed version of the extended Cartan homotopy formula. 


\section{B.2 Chern-Weil theorem}

A well-known particular case of the homotopy formula is the Chern-Weil theorem. Setting $p=0$ in (B.8) gives

$$
\int_{\partial \Delta_{1}}\left\langle\mathcal{F}_{t}^{n+1}\right\rangle=\mathrm{d} \int_{\Delta_{1}} \mathrm{~h}_{t}\left\langle\mathcal{F}_{t}^{n+1}\right\rangle
$$

where $\mathcal{F}_{t}$ is the curvature of the connection $\mathcal{A}_{t}=t_{0} \mathcal{A}_{0}+t_{1} \mathcal{A}_{1}$ with $t_{0}+t_{1}=1$. The boundary of the simplex $\Delta_{1}=\left\langle t_{0}, t_{1}\right\rangle$ is given by $\partial\left\langle t_{0}, t_{1}\right\rangle=\left\langle t_{1}\right\rangle-\left\langle t_{0}\right\rangle$ and so the left-hand side of (B.9) becomes

$$
\int_{\partial \Delta_{1}}\left\langle\mathcal{F}_{t}^{n+1}\right\rangle=\left\langle\mathcal{F}_{1}^{n+1}\right\rangle-\left\langle\mathcal{F}_{0}^{n+1}\right\rangle
$$

Since $\left\langle\mathcal{F}_{t}^{n+1}\right\rangle$ is a symmetric polynomial we have

$$
\mathrm{h}_{t}\left\langle\mathcal{F}_{t}^{n+1}\right\rangle=(n+1)\left\langle\mathrm{h}_{t} \mathcal{F}_{t} \wedge \mathcal{F}_{t}^{n}\right\rangle
$$

and using

$$
\mathrm{h}_{t} \mathcal{F}_{t}=\mathrm{d}_{t} \mathcal{A}_{t}=\mathrm{d} t_{0} \mathcal{A}_{0}+\mathrm{d} t_{1} \mathcal{A}_{1}=\mathrm{d} t_{1}\left(\mathcal{A}_{1}-\mathcal{A}_{0}\right)
$$

we get

$$
\mathrm{h}_{t}\left\langle\mathcal{F}_{t}^{n+1}\right\rangle=(n+1)\left\langle\mathrm{d} t_{1}\left(\mathcal{A}_{1}-\mathcal{A}_{0}\right) \wedge \mathcal{F}_{t}^{n}\right\rangle .
$$

Substituting into (B.9) one arrives at the Chern-Weil theorem

$$
\left\langle\mathcal{F}_{1}^{n+1}\right\rangle-\left\langle\mathcal{F}_{0}^{n+1}\right\rangle=(n+1) \mathrm{d} \int_{0}^{1} \mathrm{~d} t\left\langle\left(\mathcal{A}_{1}-\mathcal{A}_{0}\right) \wedge \mathcal{F}_{t}^{n}\right\rangle=\mathrm{d} Q_{\mathcal{A}_{1} \leftarrow \mathcal{A}_{0}}^{(2 n+1)} .
$$

\section{B.3 Triangle equation}

The case $p=1$ yields the triangle equation (4.16). Setting $p=1$ in (B.8) gives

$$
\int_{\partial \Delta_{2}} \mathrm{~h}_{t}\left\langle\mathcal{F}_{t}^{n+1}\right\rangle=-\frac{1}{2} \mathrm{~d} \int_{\Delta_{2}} \mathrm{~h}_{t}^{2}\left\langle\mathcal{F}_{t}^{n+1}\right\rangle
$$

where $\mathcal{A}_{t}=t_{0} \mathcal{A}_{0}+t_{1} \mathcal{A}_{1}+t_{2} \mathcal{A}_{2}$ with $t_{0}+t_{1}+t_{2}=1$. Again the boundary of the simplex $\Delta_{2}=\left\langle t_{0}, t_{1}, t_{2}\right\rangle$ is given by $\partial\left\langle t_{0}, t_{1}, t_{2}\right\rangle=\left\langle t_{1}, t_{2}\right\rangle-\left\langle t_{0}, t_{2}\right\rangle+\left\langle t_{0}, t_{1}\right\rangle$ and so the left-hand side of (B.15) becomes

$$
\int_{\partial \Delta_{2}} \mathrm{~h}_{t}\left\langle\mathcal{F}_{t}^{n+1}\right\rangle=Q_{\mathcal{A}_{2} \leftarrow \mathcal{A}_{1}}^{(2 n+1)}-Q_{\mathcal{A}_{2} \leftarrow \mathcal{A}_{0}}^{(2 n+1)}+Q_{\mathcal{A}_{1} \leftarrow \mathcal{A}_{0}}^{(2 n+1)}
$$

where we have used

$$
\int_{\left\langle t_{i}, t_{j}\right\rangle} \mathrm{h}_{t}\left\langle\mathcal{F}_{t}^{n+1}\right\rangle=Q_{\mathcal{A}_{j} \leftarrow \mathcal{A}_{i}}^{(2 n+1)}
$$

Using the symmetry of the invariant polynomial $\langle-\rangle$ one derives

$$
\frac{1}{2} \mathrm{~h}_{t}^{2}\left\langle\mathcal{F}_{t}^{n+1}\right\rangle=\frac{1}{2} n(n+1)\left\langle\left(\mathrm{d}_{t} \mathcal{A}_{t}\right)^{2} \wedge \mathcal{F}_{t}^{n+1}\right\rangle
$$

where

$$
\mathrm{d}_{t} \mathcal{A}_{t}=\mathrm{d} t_{0} \mathcal{A}_{0}+\mathrm{d} t_{1} \mathcal{A}_{1}+\mathrm{d} t_{2} \mathcal{A}_{2}=\mathrm{d} t_{0}\left(\mathcal{A}_{0}-\mathcal{A}_{1}\right)+\mathrm{d} t_{2}\left(\mathcal{A}_{2}-\mathcal{A}_{1}\right)
$$


Substituting into (B.18) gives

$$
\frac{1}{2} \mathrm{~h}_{t}^{2}\left\langle\mathcal{F}_{t}^{n+1}\right\rangle=-n(n+1) \mathrm{d} t_{0} \wedge \mathrm{d} t_{2}\left\langle\left(\mathcal{A}_{2}-\mathcal{A}_{1}\right) \wedge\left(\mathcal{A}_{1}-\mathcal{A}_{0}\right) \wedge \mathcal{F}_{t}^{n+1}\right\rangle .
$$

We redefine the simplex parameterization as $t=1-t_{0}, s=t_{2}$ and integrate explicitly over $\Delta_{2}$. In this way we get

$$
\frac{1}{2} \int_{\partial \Delta_{2}} \mathrm{~h}_{t}^{2}\left\langle\mathcal{F}_{t}^{n+1}\right\rangle=Q_{\mathcal{A}_{2} \leftarrow \mathcal{A}_{1} \leftarrow \mathcal{A}_{0}}^{(2 n)}
$$

where $Q_{\mathcal{A}_{2} \leftarrow \mathcal{A}_{1} \leftarrow \mathcal{A}_{0}}^{(2 n)}$ is defined in (4.17). Substituting this expression together with (B.16) into (B.15) we arrive finally at the triangle equation (4.16).

\section{$\mathrm{C} \quad \mathrm{SU}(2,2 \mid N)$ Chern-Simons supergravity}

\section{C.1 Supergravity Lagrangian}

The supersymmetric extension of the AdS algebra in five dimensions is the Lie superalgebra $\mathfrak{s u}(2,2 \mid N)[25]$. The associated gauge field decomposes into generators as

$$
A=e^{a} \mathrm{P}_{a}+\frac{1}{2} \omega^{a b} \mathrm{~J}_{a b}+a_{n}^{m} \mathrm{M}_{m}^{n}+b \mathrm{~K}+\bar{\psi}_{\alpha}^{k} \mathrm{Q}_{k}^{\alpha}-\overline{\mathrm{Q}}_{\beta}^{k} \psi_{k}^{\beta} .
$$

Here the generators $\left\{\mathrm{P}_{a}, \mathrm{~J}_{a b}\right\}$ span an $\mathfrak{s o}(4,2)$ subalgebra, $\mathrm{M}_{m}^{n}$ are $N^{2}-1$ generators of $\mathrm{SU}(N), \mathrm{K}$ generates a $\mathrm{U}(1)$ subgroup, and $\mathrm{Q}_{k}^{\alpha}, \overline{\mathrm{Q}}_{\beta}^{k}$ are the supersymmetry generators. The Chern-Simons Lagrangian associated to this superalgebra is given by $[23,25,40]$

$$
L_{\mathrm{CS}}^{(5)}=L_{\psi}+L_{a}+L_{b}+L_{e}
$$

where

$$
\begin{aligned}
& L_{\psi}=\frac{3}{2 \mathrm{i}}\left(\bar{\psi}^{n} \wedge \mathcal{R} \wedge \nabla \psi_{n}+\bar{\psi}^{n} \wedge \mathcal{F}_{n}^{m} \wedge \nabla \psi_{m}-\nabla \bar{\psi}^{n} \wedge \mathcal{R} \wedge \psi_{n}-\nabla \bar{\psi}^{n} \wedge \mathcal{F}_{n}^{m} \wedge \psi_{m}\right) \\
& L_{a}=\frac{3}{N} \mathrm{~d} b \wedge \operatorname{Tr}\left(a \wedge \mathrm{d} a+\frac{2}{3} a^{3}\right)-\mathrm{i} \operatorname{Tr}\left(a \wedge(\mathrm{d} a)^{2}+\frac{3}{2} a^{3} \wedge \mathrm{d} a+\frac{3}{5} a^{5}\right) \\
& L_{b}=\left(\frac{1}{16}-\frac{1}{N^{2}}\right) b \wedge(\mathrm{d} b)^{2}-\frac{3}{4 l^{2}} b \wedge\left(T^{a} \wedge T_{a}-R_{a b} \wedge e^{a} \wedge e^{b}-\frac{l^{2}}{2} R^{a b} \wedge R_{a b}\right) \\
& L_{e}=\frac{3}{8 l} \epsilon_{a b c d h}\left(R^{a b} \wedge R^{c d}+\frac{2}{3} R^{a b} \wedge e^{c} \wedge e^{d}+\frac{1}{5} e^{a} \wedge e^{b} \wedge e^{c} \wedge e^{d}\right) \wedge e^{h}
\end{aligned}
$$

and

$$
\begin{aligned}
\mathcal{R}=\mathrm{i} & \left(\frac{1}{4}+\frac{1}{N}\right)\left(\mathrm{d} b+\frac{\mathrm{i}}{2 l} \bar{\psi}^{n} \wedge \psi_{n}\right)+\frac{1}{2}\left(T^{a}-\frac{1}{4} \bar{\psi}^{n} \wedge \Gamma^{a} \psi_{n}\right) \Gamma_{a} \\
& +\frac{1}{4}\left(R^{a b}+\frac{1}{l} e^{a} \wedge e^{b}+\frac{1}{4 l} \bar{\psi}^{n} \wedge \Gamma^{a b} \psi_{n}\right) \Gamma_{a b}, \\
\mathcal{F}_{n}^{m}= & f_{n}^{m}-\frac{1}{2 l} \bar{\psi}^{m} \wedge \psi_{n} .
\end{aligned}
$$


Here the spinor covariant derivatives are defined by

$$
\begin{aligned}
& \nabla \psi_{k}=\mathrm{d} \psi_{k}+\frac{1}{2 l} e^{a} \wedge \Gamma_{a} \psi_{k}+\frac{1}{4} \omega^{a b} \wedge \Gamma_{a b} \psi_{k}-a_{k}^{n} \wedge \psi_{n}+\mathrm{i}\left(\frac{1}{4}-\frac{1}{N}\right) b \wedge \psi_{k} \\
& \nabla \bar{\psi}^{k}=\mathrm{d} \bar{\psi}^{k}-\frac{1}{2 l} e^{a} \wedge \bar{\psi}^{k} \Gamma_{a}-\frac{1}{4} \omega^{a b} \wedge \bar{\psi}^{k} \Gamma_{a b}+a_{n}^{k} \wedge \bar{\psi}^{n}-\mathrm{i}\left(\frac{1}{4}-\frac{1}{N}\right) b \wedge \bar{\psi}^{k}
\end{aligned}
$$

while $f=\mathrm{d} a+a \wedge a$ is the curvature of the $\mathrm{SU}(N)$ gauge field $a$. The (super)symmetry transformations and field equations can be read off from the general expressions (4.11) and (4.12) respectively.

\section{C.2 Representation of $\mathfrak{s u}(2,2 \mid 1)$}

For simplicity we consider now the particular instance $N=1$. This case furnishes the minimum number $\mathcal{N}=2$ of supersymmetries, and the commutation relations are given by

$$
\begin{aligned}
{\left[\mathrm{K}, \mathrm{Q}^{\rho}\right] } & =\frac{3 \mathrm{i}}{4} \mathrm{Q}^{\rho}, \\
{\left[\mathrm{K}, \overline{\mathrm{Q}}_{\rho}\right] } & =-\frac{3 \mathrm{i}}{4} \overline{\mathrm{Q}}_{\rho}, \\
{\left[\mathrm{P}_{a}, \mathrm{P}_{b}\right] } & =\frac{1}{l^{2}} \mathrm{~J}_{a b}, \\
{\left[\mathrm{P}_{a}, \mathrm{~J}_{b c}\right] } & =\eta_{b a} \mathrm{P}_{c}-\eta_{a c} \mathrm{P}_{b}, \\
{\left[\mathrm{P}_{a}, \mathrm{Q}^{\rho}\right] } & =-\frac{1}{2 l}\left(\Gamma_{a}\right)_{\gamma}^{\rho} \mathrm{Q}^{\gamma}, \\
{\left[\mathrm{P}_{a}, \overline{\mathrm{Q}}_{\rho}\right] } & =\frac{1}{2 l} \overline{\mathrm{Q}}_{\gamma}\left(\Gamma_{a}\right)_{\rho}^{\gamma}, \\
{\left[\mathrm{J}_{a b}, \mathrm{~J}_{c d}\right] } & =\eta_{c b} \mathrm{~J}_{a d}-\eta_{a c} \mathrm{~J}_{b d}+\eta_{d b} \mathrm{~J}_{c a}-\eta_{a d} \mathrm{~J}_{c b}, \\
{\left[\mathrm{~J}_{a b}, \mathrm{Q}^{\rho}\right] } & =-\frac{1}{2}\left(\Gamma_{a b}\right)_{\gamma}^{\rho} \mathrm{Q}^{\gamma}, \\
{\left[\mathrm{J}_{a b}, \overline{\mathrm{Q}}_{\rho}\right] } & =\frac{1}{2} \overline{\mathrm{Q}}_{\gamma}\left(\Gamma_{a b}\right)_{\rho}^{\gamma}, \\
\left\{\mathrm{Q}^{\rho}, \overline{\mathrm{Q}}_{\sigma}\right\} & =-4 \mathrm{i} \delta_{\sigma}^{\rho} \mathrm{K}_{+}+2\left(\Gamma^{a}\right)_{\sigma}^{\rho} \mathrm{P}_{a}-\left(\Gamma_{a b}\right)_{\sigma}^{\rho} \mathrm{J}_{a b} .
\end{aligned}
$$

According to (5.18)-(5.22) the matrix generators explicitly read as

$$
\begin{aligned}
\Gamma_{0}=\left(\begin{array}{cccc}
0 & \mathrm{i} & 0 & 0 \\
\mathrm{i} & 0 & 0 & 0 \\
0 & 0 & 0 & -\mathrm{i} \\
0 & 0 & -\mathrm{i} & 0
\end{array}\right), & \Gamma_{1}=\left(\begin{array}{cccc}
0 & -\mathrm{i} & 0 & 0 \\
\mathrm{i} & 0 & 0 & 0 \\
0 & 0 & 0 & \mathrm{i} \\
0 & 0 & -\mathrm{i} & 0
\end{array}\right), \\
\Gamma_{3}=\left(\begin{array}{cccc}
0 & 0 & 1 & 0 \\
0 & 0 & 0 & 1 \\
1 & 0 & 0 & 0 \\
0 & 1 & 0 & 0
\end{array}\right), & \Gamma_{4}=\left(\begin{array}{cccc}
0 & 0 & -\mathrm{i} c & 0 \\
0 & 0 & 0 & -\mathrm{i} \\
\mathrm{i} & 0 & 0 & 0 \\
0 & \mathrm{i} & 0 & 0
\end{array}\right),
\end{aligned}
$$


and using (5.2) we find

$$
\begin{aligned}
& \Gamma_{01}=\left(\begin{array}{cccc}
-1 & 0 & 0 & 0 \\
0 & 1 & 0 & 0 \\
0 & 0 & -1 & 0 \\
0 & 0 & 0 & 1
\end{array}\right), \quad \Gamma_{02}=\left(\begin{array}{cccc}
0 & -\mathrm{i} & 0 & 0 \\
\mathrm{i} & 0 & 0 & 0 \\
0 & 0 & 0 & -\mathrm{i} \\
0 & 0 & \mathrm{i} & 0
\end{array}\right), \quad \Gamma_{03}=\left(\begin{array}{cccc}
0 & 0 & 0 & \mathrm{i} \\
0 & 0 & \mathrm{i} & 0 \\
0 & -\mathrm{i} & 0 & 0 \\
-\mathrm{i} & 0 & 0 & 0
\end{array}\right), \\
& \Gamma_{04}=\left(\begin{array}{cccc}
0 & 0 & 0 & 1 \\
0 & 0 & 1 & 0 \\
0 & 1 & 0 & 0 \\
1 & 0 & 0 & 0
\end{array}\right), \quad \Gamma_{12}=\left(\begin{array}{cccc}
0 & \mathrm{i} & 0 & 0 \\
\mathrm{i} & 0 & 0 & 0 \\
0 & 0 & 0 & \mathrm{i} \\
0 & 0 & \mathrm{i} & 0
\end{array}\right), \quad \Gamma_{13}=\left(\begin{array}{cccc}
0 & 0 & 0 & -\mathrm{i} \\
0 & 0 & \mathrm{i} & 0 \\
0 & \mathrm{i} & 0 & 0 \\
-\mathrm{i} & 0 & 0 & 0
\end{array}\right), \\
& \Gamma_{14}=\left(\begin{array}{cccc}
0 & 0 & 0 & -1 \\
0 & 0 & 1 & 0 \\
0 & -1 & 0 & 0 \\
1 & 0 & 0 & 0
\end{array}\right), \quad \Gamma_{23}=\left(\begin{array}{cccc}
0 & 0 & 1 & 0 \\
0 & 0 & 0 & -1 \\
-1 & 0 & 0 & 0 \\
0 & 1 & 0 & 0
\end{array}\right), \quad \Gamma_{24}=\left(\begin{array}{cccc}
0 & 0 & -\mathrm{i} & 0 \\
0 & 0 & 0 & \mathrm{i} \\
-\mathrm{i} & 0 & 0 & 0 \\
0 & \mathrm{i} & 0 & 0
\end{array}\right), \\
& \Gamma_{34}=\left(\begin{array}{cccc}
\mathrm{i} & 0 & 0 & 0 \\
0 & \mathrm{i} & 0 & 0 \\
0 & 0 & -\mathrm{i} & 0 \\
0 & 0 & 0 & -\mathrm{i}
\end{array}\right)
\end{aligned}
$$

It is then easy to show that this particular choice of basis for the Lie algebra $\mathfrak{s u}(2,2)$ has traceless generators all satisfying the Clifford algebra relations (5.1).

The $\mathfrak{s u}(2,2 \mid 1)$-invariant tensor of rank three can be computed from this representation as the supersymmetrized supertraces of products of triples of supermatrices. The nonvanishing components are given by [23]

$$
\begin{aligned}
\left\langle\mathrm{J}_{a b} \mathrm{~J}_{c d} \mathrm{P}_{e}\right\rangle & =-\frac{\gamma}{2 l} \epsilon_{a b c d e}, \\
\langle\mathrm{KKK}\rangle & =-\frac{15}{16} \\
\left\langle\mathrm{~K} \mathrm{P}_{a} \mathrm{P}_{b}\right\rangle & =-\frac{1}{4 l^{2}} \delta_{a b}, \\
\left\langle\mathrm{~J}_{a b} \mathrm{~K} \mathrm{~J}_{c d}\right\rangle & =-\frac{1}{4}\left(\delta_{a d} \delta_{b c}+\delta_{a c} \delta_{b d}\right), \\
\left\langle\mathrm{Q}^{\alpha} \mathrm{K} \overline{\mathrm{Q}}_{\beta}\right\rangle & =\frac{5}{2 l} \delta_{\beta}^{\alpha}, \\
\left\langle\mathrm{Q}^{\alpha} \mathrm{P}_{a} \overline{\mathrm{Q}}_{\beta}\right\rangle & =-\frac{\mathrm{i}}{l}\left(\Gamma_{a}\right)_{\beta}^{\alpha}, \\
\left\langle\mathrm{Q}^{\alpha} \mathrm{J}_{a b} \overline{\mathrm{Q}}_{\beta}\right\rangle & =-\frac{\mathrm{i}}{l}\left(\Gamma_{a b}\right)_{\beta}^{\alpha},
\end{aligned}
$$

where $\gamma$ is an arbitrary constant.

Open Access. This article is distributed under the terms of the Creative Commons Attribution License (CC-BY 4.0), which permits any use, distribution and reproduction in any medium, provided the original author(s) and source are credited. 


\section{References}

[1] P. Forgacs and N.S. Manton, Spacetime symmetries in gauge theories, Commun. Math. Phys. 72 (1980) 15 [INSPIRE].

[2] D. Kapetanakis and G. Zoupanos, Coset space dimensional reduction of gauge theories, Phys. Rept. 219 (1992) 4 [INSPIRE].

[3] L. Álvarez-Consul and O. Garcia-Prada, Dimensional reduction and quiver bundles, math/0112160 [INSPIRE].

[4] O. Lechtenfeld, A.D. Popov and R.J. Szabo, Quiver gauge theory and noncommutative vortices, Prog. Theor. Phys. Suppl. 171 (2007) 258 [arXiv:0706.0979] [InSPIRE].

[5] B.P. Dolan and R.J. Szabo, Equivariant dimensional reduction and quiver gauge theories, Gen. Rel. Grav. 43 (2010) 2453 [arXiv: 1001.2429] [InSPIRE].

[6] L. Álvarez-Consul and O. Garcia-Prada, Dimensional reduction, SL(2, C) equivariant bundles and stable holomorphic chains, Int. J. Math. 12 (2001) 159 [math/0112159] [INSPIRE].

[7] O. Lechtenfeld, A.D. Popov and R.J. Szabo, Rank two quiver gauge theory, graded connections and noncommutative vortices, JHEP 09 (2006) 054 [hep-th/0603232] [INSPIRE].

[8] A.D. Popov, Integrability of vortex equations on Riemann surfaces, Nucl. Phys. B 821 (2009) 452 [arXiv:0712.1756] [InSPIRE].

[9] O. Lechtenfeld, A.D. Popov and R.J. Szabo, SU(3)-equivariant quiver gauge theories and nonabelian vortices, JHEP 08 (2008) 093 [arXiv:0806.2791] [INSPIRE].

[10] A.D. Popov and R.J. Szabo, Double quiver gauge theory and nearly Kähler flux compactifications, JHEP 02 (2012) 033 [arXiv:1009.3208] [INSPIRE].

[11] A.D. Popov, Bounces/dyons in the plane wave matrix model and $\mathrm{SU}(N)$ Yang-Mills theory, Mod. Phys. Lett. A 24 (2009) 349 [arXiv:0804.3845] [InSPIRE].

[12] N.S. Manton and N.A. Rink, Geometry and energy of nonabelian vortices, J. Math. Phys. 52 (2011) 043511 [arXiv: 1012.3014] [InSPIRE].

[13] A.D. Popov, Nonabelian vortices on Riemann surfaces: An integrable case, Lett. Math. Phys. 84 (2008) 139 [arXiv:0801.0808] [InSPIRE].

[14] A.D. Popov and R.J. Szabo, Quiver gauge theory of nonabelian vortices and noncommutative instantons in higher dimensions, J. Math. Phys. 47 (2006) 012306 [hep-th/0504025] [INSPIRE].

[15] B.P. Dolan and R.J. Szabo, Dimensional reduction, monopoles and dynamical symmetry breaking, JHEP 03 (2009) 059 [arXiv:0901.2491] [INSPIRE].

[16] M. Temple-Raston, The reduction of five-dimensional Chern-Simons theories, J. Math. Phys. 35 (1994) 759 [inSPIRE].

[17] J.P. Harnad, S. Shnider and J. Tafel, Group actions on principal bundles and dimensional reduction, Lett. Math. Phys. 4 (1980) 107.

[18] M. Nakahara, Geometry, Topology and Physics, Institute of Physics Publishing, (2003).

[19] J.A. de Azcarraga and J.M. Izquierdo, Lie Groups, Lie Algebras, Cohomology and Some Applications in Physics, Cambridge University Press, (1995).

[20] A. Borowiec, M. Ferraris and M. Francaviglia, A covariant formalism for Chern-Simons gravity, J. Phys. A 36 (2003) 2589 [hep-th/0301146] [InSPIRE]. 
[21] P. Mora, R. Olea, R. Troncoso and J. Zanelli, Transgression forms and extensions of Chern-Simons gauge theories, JHEP 02 (2006) 067 [hep-th/0601081] [INSPIRE].

[22] F. Izaurieta, E. Rodriguez and P. Salgado, On transgression forms and Chern-Simons (super)gravity, hep-th/0512014 [INSPIRE].

[23] F. Izaurieta, E. Rodriguez and P. Salgado, The extended Cartan homotopy formula and a subspace separation method for Chern-Simons supergravity, Lett. Math. Phys. 80 (2007) 127 [hep-th/0603061] [INSPIRE].

[24] J. Manes, R. Stora and B. Zumino, Algebraic study of chiral anomalies, Commun. Math. Phys. 102 (1985) 157 [InSPIRE].

[25] A.H. Chamseddine, Topological gravity and supergravity in various dimensions, Nucl. Phys. B 346 (1990) 213 [inSPIRE].

[26] J.A. de Azcarraga, A.J. Macfarlane, A.J. Mountain and J.C. Perez Bueno, Invariant tensors for simple groups, Nucl. Phys. B 510 (1998) 657 [physics/9706006] [INSPIRE].

[27] S. Deser, R. Jackiw and S. Templeton, Topologically massive gauge theories, Annals Phys. 140 (1982) 372 [Erratum ibid. 185 (1988) 406] [INSPIRE].

[28] M. Bañados, L.J. Garay and M. Henneaux, The local degrees of freedom of higher dimensional pure Chern-Simons theories, Phys. Rev. D 53 (1996) 593 [hep-th/9506187] [InSPIRE].

[29] M. Bañados, L.J. Garay and M. Henneaux, The dynamical structure of higher dimensional Chern-Simons theory, Nucl. Phys. B 476 (1996) 611 [hep-th/9605159] [InSPIRE].

[30] O. Mišković, R. Troncoso and J. Zanelli, Canonical sectors of five-dimensional Chern-Simons theories, Phys. Lett. B 615 (2005) 277 [hep-th/0504055] [INSPIRE].

[31] J. Saavedra, R. Troncoso and J. Zanelli, Degenerate dynamical systems, J. Math. Phys. 42 (2001) 4383 [hep-th/0011231] [InSPIRE].

[32] O. Mišković and J. Zanelli, Dynamical structure of irregular constrained systems, J. Math. Phys. 44 (2003) 3876 [hep-th/0302033] [INSPIRE].

[33] D. Lovelock, The Einstein tensor and its generalizations, J. Math. Phys. 12 (1971) 498 [INSPIRE].

[34] C. Lanczos, A remarkable property of the Riemann-Christoffel tensor in four dimensions, Annals Math. 39 (1938) 842 [INSPIRE].

[35] R. Zegers, Birkhoff's theorem in Lovelock gravity, J. Math. Phys. 46 (2005) 072502 [gr-qc/0505016] [INSPIRE].

[36] A.H. Chamseddine, Topological gauge theory of gravity in five-dimensions and all odd dimensions, Phys. Lett. B 233 (1989) 291 [INSPIRE].

[37] J. Zanelli, Lecture notes on Chern-Simons (super-)gravities. Second edition (February 2008), hep-th/0502193 [INSPIRE].

[38] G. Allemandi, M. Francaviglia and M. Raiteri, Charges and energy in Chern-Simons theories and Lovelock gravity, Class. Quant. Grav. 20 (2003) 5103 [gr-qc/0308019] [INSPIRE].

[39] R. Troncoso and J. Zanelli, Higher dimensional gravity, propagating torsion and AdS gauge invariance, Class. Quant. Grav. 17 (2000) 4451 [hep-th/9907109] [INSPIRE].

[40] R. Troncoso and J. Zanelli, Gauge supergravities for all odd dimensions, Int. J. Theor. Phys. 38 (1999) 1181 [hep-th/9807029] [INSPIRE]. 
[41] M. Bañados, R. Troncoso and J. Zanelli, Higher dimensional Chern-Simons supergravity, Phys. Rev. D 54 (1996) 2605 [gr-qc/9601003] [INSPIRE].

[42] R. Troncoso and J. Zanelli, Chern-Simons supergravities with off-shell local superalgebras, proceedings of Black Holes and the Structure of the Universe, C. Teitelboim and J. Zanelli eds., World Scientific (2000), pg. 119-145, [hep-th/9902003] [INSPIRE].

[43] M. Bañados, Gravitons and gauge fields in Chern-Simons supergravity, Nucl. Phys. Proc. Suppl. 88 (2000) 17 [hep-th/9911150] [InSPIRE].

[44] A. Bouchareb, C.-M. Chen, G. Clément and D.V. Gal'tsov, Bertotti-Robinson solutions of $D=5$ Einstein-Maxwell-Chern-Simons-Lambda theory, Phys. Rev. D 88 (2013) 084048 [arXiv: 1308.6461] [INSPIRE].

[45] A. Campoleoni, S. Fredenhagen, S. Pfenninger and S. Theisen, Asymptotic symmetries of three-dimensional gravity coupled to higher-spin fields, JHEP 11 (2010) 007 [arXiv: 1008.4744] [INSPIRE].

[46] S.L. Cacciatori, M.M. Caldarelli, A. Giacomini, D. Klemm and D.S. Mansi, Chern-Simons formulation of three-dimensional gravity with torsion and nonmetricity, J. Geom. Phys. 56 (2006) 2523 [hep-th/0507200] [INSPIRE]. 